\title{
THE INVERSE STURM-LIOUVILLE PROBLEM WITH MIXED BOUNDARY CONDITIONS
}

\author{
E. KOROTYAEV AND D. CHELKAK
}

\begin{abstract}
Let $H \psi=-\psi^{\prime \prime}+q \psi, \psi(0)=0, \psi^{\prime}(1)+b \psi(1)=0$ be a selfadjoint SturmLiouville operator acting in $L^{2}(0,1)$. Let $\lambda_{n}(q, b)$ and $\nu_{n}(q, b)$ denote its eigenvalues and the so-called norming constants, respectively. A complete characterization of all spectral data $\left(\left\{\lambda_{n}\right\}_{n=0}^{+\infty} ;\left\{\nu_{n}\right\}_{n=0}^{+\infty}\right)$ corresponding to $(q ; b) \in L^{2}(0,1) \times \mathbb{R}$ is given, together with a similar characterization for fixed $b$ and a parametrization of isospectral manifolds.
\end{abstract}

\section{$\S 1$. InTRODUCTION AND THE MAIN RESUlts}

We consider the Sturm-Liouville problem

$$
\begin{gathered}
-\psi^{\prime \prime}(x)+q(x) \psi(x)=\lambda \psi(x), \quad x \in[0,1], \\
\psi^{\prime}(0)-a \psi(0)=0, \quad \psi^{\prime}(1)+b \psi(1)=0, \quad a, b \in \mathbb{R} \cup\{\infty\},
\end{gathered}
$$

where $q$ is a real potential belonging to the space $L^{2}(0,1)$, i.e., $\|q\|^{2}=\int_{0}^{1}|q(t)|^{2} d t<+\infty$.

The inverse spectral theory for Sturm-Liouville operators on a finite interval was investigated by many authors (G. Borg, I. M. Gel'fand, B. M. Levitan, V. A. Marchenko, E. Trubowitz, ...); see the monographs Lev84, Mar77, PT87. In general, the inverse spectral problem consists of the following parts.

(i) The uniqueness theorem, i.e., the fact that the spectral data (eigenvalues and, usually, some "additional" parameters) determine the potential uniquely.

(ii) The reconstruction procedure (recovering the potential by the spectral data).

(iii) Characterization (description of all spectral data corresponding to a fixed class of potentials).

The first uniqueness theorems were obtained by Ambarzumyan Am29 and Borg [Bo46. The first reconstruction procedure was given by Gel'fand and Levitan GL51] (see the details in Lev84, Mar77]). Later, Trubowitz and his coauthors (IT83, IMT84, DT84, (PT87]) developed an "analytic" approach to the characterization problem, based on the smoothness of the mapping \{potentials\} $\mapsto\{$ spectral data $\}$ and on an explicit reconstruction procedure for the special case where only one spectral datum is changed. In particular, the solution of the inverse Dirichlet (i.e., $a=b=\infty$ ) problem on $[0,1]$ via this approach was given in the book PT87. In [T83, the case where (not fixed) $a, b$ run through $\mathbb{R}$ was considered (we recall the main result of IT83 below; see Theorem [5.1), and the paper IMT84 was devoted to the case of fixed $a, b \in \mathbb{R}$. We note that in IT83, IMT84 the analysis of the mapping $(q, a, b) \mapsto\{$ spectral data $\}$ was reduced to the analysis of the Dirichlet problem (by using the Darboux transform, which adds the

2010 Mathematics Subject Classification. Primary 34B24.

Key words and phrases. Inverse spectral theory, Sturm-Liouville operators.

The first author was partially supported by EPSRC, grant EP/D054621.

The second author was partially supported by the Foundation of the President of the Russian Federation (grants no. MK-4306.2008.1 and NSh-2409.2008.1). 
ground eigenvalue). This reduction makes it possible to prove the characterization theorem, to describe the isospectral manifolds (i.e., the set of all $(q, a, b)$ with fixed spectrum), etc.

Let $a=\infty, b \in \mathbb{R}$. Below we describe the results of Dahlberg and Trubowitz DT84] (as far as we know, in the case in question this is the most detailed characterization of spectral data available in the literature). Let

$$
H \psi=-\psi^{\prime \prime}+q(x) \psi, \quad \psi(0)=0, \quad \psi^{\prime}(1)+b \psi(1)=0, \quad b \in \mathbb{R} .
$$

We denote by $\left\{\lambda_{n}(q, b)\right\}_{n=0}^{+\infty}$ the eigenvalues of $H$. It is well known that all $\lambda_{n}(q, b)$ are simple and $\lambda_{n}(q, b)=\lambda_{n}^{0}+Q_{0}+2 b+\mu_{n}(q, b)$, where $\left\{\mu_{n}(q, b)\right\}_{n=0}^{+\infty} \in \ell^{2}=\ell_{0}^{2}$ and $Q_{0}=\int_{0}^{1} q(t) d t$. Here and below, $\lambda_{n}^{0}=\pi^{2}\left(n+\frac{1}{2}\right)^{2}, n \geq 0$, denote the unperturbed eigenvalues and

$$
\ell_{k}^{2}=\left\{\left\{f_{n}\right\}_{n=0}^{+\infty}: \sum_{n=0}^{+\infty}\left|(n+1)^{k} f_{n}\right|^{2}<+\infty\right\} .
$$

The monotonicity property $\lambda_{0}<\lambda_{1}<\cdots$ implies that, even if $(q ; b)$ runs through $L^{2}(0,1) \times \mathbb{R},\left\{\mu_{n}\right\}_{n=0}^{+\infty}$ fails to run through the entire space $\ell^{2}$. Let

$$
\mathcal{M}=\left\{\left\{\mu_{n}\right\}_{n=0}^{+\infty} \in \ell^{2}: \lambda_{0}^{0}+\mu_{0}<\lambda_{1}^{0}+\mu_{1}<\cdots\right\} \subset \ell^{2} .
$$

The main results of [DT84] are as follows.

(i) A sequence of real numbers $\lambda_{n}^{*}=\lambda_{n}^{0}+c^{*}+\mu_{n}^{*}, c^{*} \in \mathbb{R}$, is the spectrum of $H$ for some potential $q \in L^{2}(0,1)$ if and only if $\left\{\mu_{n}^{*}\right\}_{n=0}^{+\infty} \in \mathcal{M}$.

(ii) Suppose $q \in L^{2}(0,1)$ and $e \in L_{\text {even }}^{2}(0,1)$, i.e., $e(1-x) \equiv e(x), x \in[0,1]$. Two cases are possible:

(a) there is no potential $p \in L^{2}(0,1)$ such that the Dirichlet spectrum of $p$ coincides with the Dirichlet spectrum of $e$ and $\lambda_{n}(p, b)=\lambda_{n}(q, b)$ for all $n \geq 0$;

(b) such a $p$ exists and is unique.

(iii) An explicit condition that distinguishes cases (a) and (b) from each other is given in terms of $\left\{\lambda_{n}(q, b)\right\}_{n=0}^{+\infty}$ and the Dirichlet spectrum of e.

Roughly speaking, (ii) describes a (smooth) bijection between the isospectral set

$$
\mathrm{IsO}_{b}\left[\left\{\lambda_{n}^{*}\right\}_{n=0}^{+\infty}\right]=\left\{q \in L^{2}(0,1):\left\{\lambda_{n}(q, b)\right\}_{n=0}^{+\infty}=\left\{\lambda_{n}^{*}\right\}_{n=0}^{+\infty}\right\}
$$

and some open subset of $L_{\text {even }}^{2}(0,1)$ (see DT84 for the details). Our main goals in the present paper are to give a more explicit characterization of spectral data in the style of [PT87], IT83] and to parametrize the isospectral manifolds for fixed $b$ in a more "classical" way.

Let $\varphi(x)=\varphi(x, \lambda, q), \xi_{b}(x)=\xi_{b}(x, \lambda, q)$ be the solutions of the equation $-\psi^{\prime \prime}+$ $q(x) \psi=\lambda \psi$ such that

$$
\varphi(0)=0, \quad \varphi^{\prime}(0)=1 \quad \text { and } \quad \xi_{b}(1)=-1, \quad \xi_{b}^{\prime}(1)=b
$$

(here and below we denote $\left({ }^{\prime}\right)=\frac{\partial}{\partial x}$ and $\left.\left({ }^{\cdot}\right)=\frac{\partial}{\partial \lambda}\right)$ ). Note that the $\lambda_{n}(q, b)$ are the roots of the Wronskian

$$
w(\lambda)=w(\lambda, q, b):=\left\{\varphi, \xi_{b}\right\}(\lambda, q) \equiv \varphi^{\prime}(1, \lambda, q)+b \varphi(1, \lambda, q), \quad \lambda \in \mathbb{C},
$$

where $\left\{\varphi, \xi_{b}\right\}=\varphi \xi_{b}^{\prime}-\varphi^{\prime} \xi_{b}$. The Hadamard factorization theorem implies that

$$
w(\lambda, q, b) \equiv \cos \sqrt{\lambda} \cdot \prod_{n=0}^{+\infty} \frac{\lambda-\lambda_{n}(q, b)}{\lambda-\lambda_{n}^{0}}, \quad \lambda \in \mathbb{C} .
$$


Let $\psi_{n}(x)=\psi_{n}(x, q, b)$ be the $n$th normalized eigenfunction of $H$ such that $\psi_{n}^{\prime}(0)>0$. We introduce the norming constants ("additional" spectral data) as

$$
\nu_{n}(q, b)=\log \left[(-1)^{n} \varphi\left(1, \lambda_{n}(q, b), q\right)\right]=\log \left|\frac{\psi_{n}(1, q, b)}{\psi_{n}^{\prime}(0, q, b)}\right|, \quad n \geq 0 .
$$

Then $\left\|\varphi\left(\cdot, \lambda_{n}, q\right)\right\|^{2}=(-1)^{n+1} e^{\nu_{n}(q, b)} \dot{w}\left(\lambda_{n}, q, b\right)$ (see Lemma 2.1). A simple calculation gives

$$
\nu_{n}^{0}=\nu_{n}(0,0)=-\log k_{n}^{0}, \quad \text { where } \quad k_{n}^{0}=\sqrt{\lambda_{n}^{0}}=\pi\left(n+\frac{1}{2}\right) .
$$

Recall that $\lambda_{n}(q, b)=\lambda_{n}^{0}+Q_{0}+2 b+\mu_{n}(q, b)$.

Theorem 1.1. (i) The mapping

$$
\Phi:(q ; b) \mapsto\left(Q_{0}+2 b ;\left\{\mu_{n}(q, b)\right\}_{n=0}^{+\infty} ;\left\{\nu_{n}(q, b)-\nu_{n}^{0}\right\}_{n=0}^{+\infty}\right)
$$

is a real-analytic isomorphism between $L^{2}(0,1) \times \mathbb{R}$ and $\mathbb{R} \times \mathcal{M} \times \ell_{1}^{2}$.

(ii) For each $(q ; b) \in L^{2}(0,1) \times \mathbb{R}$, the following identity holds true:

$$
b=\sum_{n=0}^{+\infty}\left(2-\frac{e^{\nu_{n}(q, b)}}{\left|\dot{w}\left(\lambda_{n}, q, b\right)\right|}\right) .
$$

Remarks. (i) We adapt the approach from PT87. Its main ingredients are the smoothness of the (nonlinear) mapping \{potentials $\rightarrow$ \{spectral data $\}$ and an explicit reconstruction procedure in the case where only one $\lambda_{n}$ or $\nu_{n}$ is changed. By using essentially the same arguments, characterization theorems can be proved for other classes of potentials.

(ii) Identity (1.4) gives an explicit expression of $b$ in terms of the spectral data (if $a=\infty$ ). It can be rewritten in the form $b=\sum_{n=0}^{+\infty}\left(2-\left\|\xi_{b}\left(\cdot, \lambda_{n}, q\right)\right\|^{-2}\right)$ (see Lemma 2.1). Similar identities were proved in [JL97] with the help of the transmutation operators technique and the Gel'fand-Levitan equation. Our proof is based on simple contour integration.

(iii) Similar arguments show that the main result of [IT83, i.e., a complete characterization of the spectral data for $a, b \in \mathbb{R}$, can be reproved without reduction to the inverse Dirichlet problem. In $\S 5$ (Appendix) we derive an analog of identity (1.4) for this case.

Suppose that the "boundary parameter" $b \in \mathbb{R}$ is fixed (e.g., $b=0$ corresponds to the boundary conditions $\left.\psi(0)=0, \psi^{\prime}(1)=0\right)$. In this case, the spectral data $\left\{\lambda_{n}\right\}_{n=0}^{+\infty}$ and $\left\{\nu_{n}\right\}_{n=0}^{+\infty}$ are not independent because they satisfy the nonlinear equation (1.4). It turns out that the first eigenvalue $\lambda_{0}(q, b)$ can be uniquely recovered by the other spectral data $\left\{\lambda_{n}\right\}_{n=1}^{+\infty}$ and $\left\{\nu_{n}\right\}_{n=0}^{+\infty}$.

Corollary 1.2. For any $b \in \mathbb{R}$, the mapping

$$
\Phi_{b}: q \mapsto\left(Q_{0}+2 b ;\left\{\mu_{n}(q, b)\right\}_{n=1}^{+\infty} ;\left\{\nu_{n}(q, b)-\nu_{n}^{0}\right\}_{n=0}^{+\infty}\right)
$$

is a real-analytic isomorphism between $L^{2}(0,1)$ and $\mathbb{R} \times \mathcal{M}^{(1)} \times \ell_{1}^{2}$, where

$$
\mathcal{M}^{(1)}=\left\{\left\{\mu_{n}\right\}_{n=1}^{+\infty} \in \ell^{2}: \lambda_{1}^{0}+\mu_{1}<\lambda_{2}^{0}+\mu_{2}<\cdots\right\} \subset \ell^{2} .
$$

Instead of the first eigenvalue $\lambda_{0}$, an arbitrary norming constant $\mu_{m}$ can be "excluded" from the spectral data; this yields a parametrization of isospectral manifolds. For any $\left\{\lambda_{n}^{*}\right\}_{n=0}^{+\infty}$ such that $\lambda_{n}^{*}=\lambda_{n}^{0}+c^{*}+\mu_{n}^{*}$, where $\left(c^{*} ;\left\{\mu_{n}^{*}\right\}_{n=0}^{+\infty}\right) \in \mathbb{R} \times \mathcal{M}$, we denote

$$
w^{*}(\lambda):=\cos \sqrt{\lambda} \cdot \prod_{n=0}^{+\infty} \frac{\lambda-\lambda_{n}^{*}}{\lambda-\lambda_{n}^{0}}, \quad \lambda \in \mathbb{C} .
$$

Recall that $w(\lambda, q, b) \equiv w^{*}(\lambda)$ for all $q \in \mathrm{Iso}_{b}\left[\left\{\lambda_{n}^{*}\right\}_{n=0}^{+\infty}\right]$. 
Corollary 1.3. Suppose $b \in \mathbb{R}$ and $\left(c^{*} ;\left\{\mu_{n}^{*}\right\}_{n=0}^{+\infty}\right) \in \mathbb{R} \times \mathcal{M}$. Then for each $m \geq 0$ the mapping

$$
q \mapsto\left\{\nu_{n}(q, b)-\nu_{n}^{0}\right\}_{n=0, n \neq m}^{+\infty}
$$

is a real-analytic isomorphism between the isospectral set $\mathrm{Iso}_{b}\left[\left\{\lambda_{n}^{*}\right\}_{n=0}^{+\infty}\right]$ (which is a realanalytic submanifold of $\left.L^{2}(0,1)\right)$ and the open set

$$
\mathcal{N}_{m}^{b}=\left\{\left\{\nu_{n}-\nu_{n}^{0}\right\}_{n=0, n \neq m}^{+\infty} \in \ell_{1}^{2}: \sum_{n=0, n \neq m}^{+\infty}\left(2-\frac{e^{\nu_{n}}}{\left|\dot{w}^{*}\left(\lambda_{n}^{*}\right)\right|}\right)>b-2\right\} \subset \ell_{1}^{2} .
$$

In conclusion, note that Trubowitz's approach works well in other inverse spectral problems with a purely discrete spectrum. In particular, in our papers CKK04 and [CK07, his approach was applied to inverse problems for the 1-dimensional perturbed harmonic oscillator and the 3-dimensional harmonic oscillator perturbed by a spherically symmetric potential (reformulated for the half-line $\mathbb{R}_{+}$). In the latter case, the boundary conditions are essentially nonsymmetric; this gives an additional motivation to investigate the case of mixed conditions on $[0,1]$, which are also essentially nonsymmetric. Developing this approach, we also obtained some results concerning the characterization of spectral data for Sturm-Liouville operators with matrix potentials; see CK09.

In $\S 2$ we prove identity (1.4) and the uniqueness theorem. $\S 3$ is devoted to analytic properties of the mapping $\Phi$. In $\S 4$ we prove our main results: Theorem 1.1 and Corollaries 1.3, 1.2. $\S 5$ (Appendix) is devoted to the case where $a, b \in \mathbb{R}$.

\section{§2. Preliminaries and the proof of identity (1.4)}

Let $\vartheta(x)=\vartheta(x, \lambda, q)$ and $\varphi(x)=\varphi(x, \lambda, q)$ be the fundamental solutions of the differential equation

$$
-\psi^{\prime \prime}+q(x) \psi=\lambda \psi, \quad \lambda \in \mathbb{C},
$$

such that $\vartheta(0)=1, \vartheta^{\prime}(0)=0$ and $\varphi(0)=0, \varphi^{\prime}(0)=1$. For each $x \in[0,1]$, the functions $\vartheta, \vartheta^{\prime}, \varphi, \varphi^{\prime}$ are entire with respect to $(\lambda, q) \in \mathbb{C} \times L_{\mathbb{C}}^{2}(0,1)$ (see [PT87]). Moreover, the following asymptotics are valid:

$$
\vartheta(x, \lambda, q)=\cos \sqrt{\lambda} x+\frac{1}{2 \sqrt{\lambda}} \int_{0}^{x}(\sin \sqrt{\lambda} x+\sin \sqrt{\lambda}(x-2 t)) q(t) d t+O\left(\frac{e^{|\operatorname{Im} \sqrt{\lambda}| x}}{|\lambda|}\right),
$$

$$
\vartheta^{\prime}(x, \lambda, q)=-\sqrt{\lambda} \sin \sqrt{\lambda} x+\frac{1}{2} \int_{0}^{x}(\cos \sqrt{\lambda} x+\cos \sqrt{\lambda}(x-2 t)) q(t) d t+O\left(\frac{e^{|\operatorname{Im} \sqrt{\lambda}| x}}{|\lambda|^{1 / 2}}\right),
$$

$$
\varphi(x, \lambda, q)=\frac{\sin \sqrt{\lambda} x}{\sqrt{\lambda}}+\frac{1}{2 \lambda} \int_{0}^{x}(-\cos \sqrt{\lambda} x+\cos \sqrt{\lambda}(x-2 t)) q(t) d t+O\left(\frac{e^{|\operatorname{Im} \sqrt{\lambda}| x}}{|\lambda|^{3 / 2}}\right),
$$

$$
\varphi^{\prime}(x, \lambda, q)=\cos \sqrt{\lambda} x+\frac{1}{2 \sqrt{\lambda}} \int_{0}^{x}(\sin \sqrt{\lambda} x-\sin \sqrt{\lambda}(x-2 t)) q(t) d t+O\left(\frac{e^{|\operatorname{Im} \sqrt{\lambda}| x}}{|\lambda|}\right)
$$

as $|\lambda| \rightarrow \infty$, uniformly on bounded sets of $(x ; q) \in[0,1] \times L_{C}^{2}(0,1)$. 
Lemma 2.1. For each $(q ; b) \in L^{2}(0,1) \times \mathbb{R}$ and $n \geq 0$, we have

$$
\begin{aligned}
\left\|\varphi\left(\cdot, \lambda_{n}, q\right)\right\|^{2} & =(-1)^{n+1} e^{\nu_{n}} \dot{w}\left(\lambda_{n}, q, b\right), \\
\left\|\xi_{b}\left(\cdot, \lambda_{n}, q\right)\right\|^{2} & =(-1)^{n+1} e^{-\nu_{n}} \dot{w}\left(\lambda_{n}, q, b\right), \\
\psi_{n}^{2}(x, q, b) & \equiv \frac{\varphi\left(x, \lambda_{n}, q\right) \xi_{b}\left(x, \lambda_{n}, q\right)}{\dot{w}\left(\lambda_{n}, q, b\right)}, \quad x \in[0,1],
\end{aligned}
$$

where $\lambda_{n}=\lambda_{n}(q, b)$ and $\nu_{n}=\nu_{n}(q, b), n \geq 0$.

Proof. Let $\lambda=\lambda_{n}(q, b)$ be an eigenvalue of $H$. By the definition (1.3), we have

$$
\varphi(x) \equiv-\varphi(1) \xi_{b}(x) \equiv(-1)^{n+1} e^{\nu_{n}} \xi_{b}(x), \quad x \in[0,1] .
$$

The standard identity $\varphi^{2}=\{\dot{\varphi}, \varphi\}^{\prime}$ gives

$$
\|\varphi\|^{2}=\{\dot{\varphi}, \varphi\}(1)=(-1)^{n+1} e^{\nu_{n}}\left(\dot{\varphi} \xi_{b}^{\prime}-\dot{\varphi}^{\prime} \xi_{b}\right)(1)=(-1)^{n+1} e^{\nu_{n}} \dot{w}\left(\lambda_{n}, q, b\right),
$$

because $\dot{\varphi}(0)=\dot{\varphi}^{\prime}(0)=\dot{\xi}_{b}(1)=\dot{\xi}_{b}^{\prime}(1)=0$. Furthermore,

$$
\psi_{n}^{2}(x) \equiv \frac{\varphi^{2}(x)}{\|\varphi\|^{2}} \equiv \frac{\varphi(x) \cdot(-1)^{n+1} e^{-\nu_{n}} \varphi(x)}{\dot{w}\left(\lambda_{n}, q, b\right)} \equiv \frac{\varphi(x) \xi_{b}(x)}{\dot{w}\left(\lambda_{n}, q, b\right)},
$$

$x \in[0,1]$, and $\left\|\xi_{b}\right\|^{2}=e^{-2 \nu_{n}}\|\varphi\|^{2}=(-1)^{n+1} e^{-\nu_{n}} \dot{w}\left(\lambda_{n}, q, b\right)$.

Lemma 2.2. For each $(q ; b) \in L^{2}(0,1) \times \mathbb{R}$, we have

$$
b=\sum_{n=0}^{+\infty}\left(2-\frac{e^{\nu_{n}}}{\left|\dot{w}\left(\lambda_{n}, q, b\right)\right|}\right),
$$

where $\lambda_{n}=\lambda_{n}(q, b)$ and $\nu_{n}=\nu_{n}(q, b), n \geq 0$.

Proof. Consider the meromorphic function

$$
f(\lambda):=\frac{\varphi(1, \lambda, q)}{w(\lambda, q, b)} \equiv \frac{\varphi}{\varphi^{\prime}+b \varphi}(1, \lambda, q), \quad \lambda \in \mathbb{C} .
$$

All roots $\lambda_{n}=\lambda_{n}(q, b)$ of $w(\lambda)=w(\lambda, q, b)$ are simple, and $\operatorname{sgn} \dot{w}\left(\lambda_{n}\right)=(-1)^{n+1}$. Therefore, by (1.3),

$$
\operatorname{res}_{\lambda=\lambda_{n}} f(\lambda)=\frac{\varphi(1, \lambda, q)}{\dot{w}\left(\lambda_{n}\right)}=\frac{(-1)^{n} e^{\nu_{n}}}{\dot{w}\left(\lambda_{n}\right)}=-\frac{e^{\nu_{n}}}{\left|\dot{w}\left(\lambda_{n}\right)\right|} \quad \text { for all } n \geq 0 .
$$

Put $|\lambda|=\pi^{2} m^{2} \rightarrow \infty$. Then, using (2.4), (2.5), and the formula $\int_{0}^{1} e^{i k(1-2 t)} q(t) d t=$ $o\left(e^{|\operatorname{Im} k|}\right),|k| \rightarrow \infty$, we obtain

$$
\begin{aligned}
f(\lambda) & =\frac{\lambda^{-1 / 2} \sin \sqrt{\lambda}-\lambda^{-1} \cos \sqrt{\lambda} \cdot \frac{1}{2} Q_{0}+o\left(\lambda^{-1} e^{|\operatorname{Im} \sqrt{\lambda}|}\right)}{\cos \sqrt{\lambda}+\lambda^{-1 / 2} \sin \sqrt{\lambda} \cdot\left(\frac{1}{2} Q_{0}+b\right)+o\left(\lambda^{-1 / 2} e^{|\operatorname{Im} \sqrt{\lambda}|}\right)} \\
& =\frac{\lambda^{-1 / 2} \tan \sqrt{\lambda}-\lambda^{-1} \cdot \frac{1}{2} Q_{0}+o\left(\lambda^{-1}\right)}{1+\lambda^{-1 / 2} \tan \sqrt{\lambda} \cdot\left(\frac{1}{2} Q_{0}+b\right)+o\left(\lambda^{-1 / 2}\right)} \\
& =\lambda^{-\frac{1}{2}} \tan \sqrt{\lambda}-\lambda^{-1} \cdot \frac{1}{2} Q_{0}-\lambda^{-1} \tan ^{2} \sqrt{\lambda} \cdot\left(\frac{1}{2} Q_{0}+b\right)+o\left(\lambda^{-1}\right) .
\end{aligned}
$$

Let $f_{0}(\lambda) \equiv \lambda^{-\frac{1}{2}} \tan \sqrt{\lambda}$. An application of the Cauchy theorem in the disk $|\lambda|<\pi^{2} m^{2}$ yields

$$
\sum_{n=0}^{m}\left(\underset{\lambda=\lambda_{n}}{\operatorname{res}} f(\lambda)-\underset{\lambda=\lambda_{n}^{0}}{\operatorname{res}} f_{0}(\lambda)\right)=-\frac{1}{2} Q_{0}-\left(\frac{1}{2} Q_{0}+b\right) \cdot \sum_{n=0}^{m} \underset{\lambda=\lambda_{n}^{0}}{\operatorname{res}}\left(f_{0}(\lambda)\right)^{2}+o(1) .
$$

Observe that

$$
f_{0}(\lambda)=\frac{\tan \sqrt{\lambda}}{\sqrt{\lambda}}=-\frac{2}{\lambda-\lambda_{n}^{0}}\left(1+\frac{\lambda-\lambda_{n}^{0}}{4 \lambda_{n}^{0}}+O\left(\left(\lambda-\lambda_{n}^{0}\right)^{2}\right)\right) \quad \text { as } \quad \lambda \rightarrow \lambda_{n}^{0}, \quad n \geq 0 .
$$


Hence,

$$
\underset{\lambda=\lambda_{n}^{0}}{\operatorname{res}} f_{0}(\lambda)=-2, \quad \underset{\lambda=\lambda_{n}^{0}}{\operatorname{res}}\left(f_{0}(\lambda)\right)^{2}=-\frac{2}{\lambda_{n}^{0}}, \quad n \geq 0
$$

and

$$
\sum_{n=0}^{+\infty} \underset{\lambda=\lambda_{n}^{0}}{\operatorname{res}}\left(f_{0}(\lambda)\right)^{2}=-\frac{2}{\pi^{2}} \sum_{n=0}^{+\infty} \frac{1}{\left(n+\frac{1}{2}\right)^{2}}=-\frac{2}{\pi^{2}} \cdot \frac{\pi^{2}}{2}=-1 .
$$

Substituting these identities and (2.6) in (2.7), we obtain (1.4) as $m \rightarrow \infty$.

Theorem 2.3 (Uniqueness theorem). Let $\lambda_{n}(q, b)=\lambda_{n}(p, h)$ and $\nu_{n}(q, b)=\nu_{n}(p, h)$ for all $n \geq 0$ and some $(q ; b),(p ; h) \in L^{2}(0,1) \times \mathbb{R}$. Then $(q ; b)=(p ; h)$.

Proof. Lemma 2.2 immediately gives $b=h$. The rest of the proof is standard (see PT87, p. 58]). Define

$$
\begin{array}{ll}
f_{1}(\lambda, x):=\frac{F_{1}(\lambda, x)}{w(\lambda, q, b)}, & F_{1}(\lambda, x):=\varphi(x, \lambda, p) \xi_{b}^{\prime}(x, \lambda, q)-\xi_{b}(x, \lambda, p) \varphi^{\prime}(x, \lambda, q), \\
f_{2}(\lambda, x):=\frac{F_{2}(\lambda, x)}{w(\lambda, q, b)}, & F_{2}(\lambda, x):=\varphi(x, \lambda, p) \xi_{b}(x, \lambda, q)-\xi_{b}(x, \lambda, p) \varphi(x, \lambda, q) .
\end{array}
$$

Recall that

$$
w(\lambda, q, b) \equiv \varphi^{\prime}(1, \lambda, q)+b \varphi(1, \lambda, q) \equiv\left\{\varphi, \xi_{b}\right\}(\lambda, q), \quad \lambda \in \mathbb{C} .
$$

All roots $\lambda_{n}(q, b)=\lambda_{n}(p, b)$ of the denominator $w(\cdot, q, b)$ are simple. Moreover, they are roots of the numerators $F_{1}, F_{2}$, because (1.3) implies that

$$
\varphi\left(\cdot, \lambda_{n}, p\right)=(-1)^{n+1} e^{\nu_{n}} \xi_{b}\left(\cdot, \lambda_{n}, p\right), \quad \varphi\left(\cdot, \lambda_{n}, q\right)=(-1)^{n+1} e^{\nu_{n}} \xi_{b}\left(\cdot, \lambda_{n}, q\right) .
$$

Hence, the functions $f_{1}, f_{2}$ are entire with respect to $\lambda$ for each $x \in[0,1]$. Let $|\lambda|=\pi^{2} m^{2}$, $m \rightarrow \infty$. The asymptotics (2.4), (2.5) show that

$$
w(\lambda, q, b)=\cos \sqrt{\lambda}+O\left(|\lambda|^{-1 / 2} e^{\mid \operatorname{Im} \sqrt{\lambda \mid}}\right)=\cos \sqrt{\lambda} \cdot\left(1+O\left(|\lambda|^{-1 / 2}\right) .\right.
$$

Since

$$
\xi_{b}(x, \lambda, q) \equiv-\vartheta\left(1-x, \lambda, q^{\sharp}\right)-b \varphi\left(1-x, \lambda, q^{\sharp}\right),
$$

where $q^{\sharp}(x) \equiv q(1-x), x \in[0,1]$, the asymptotics (2.2)-(2.5) give

$$
\begin{aligned}
F_{1}(x, \lambda) & =-\sin \sqrt{\lambda} x \cdot \sin \sqrt{\lambda}(1-x)+\cos \sqrt{\lambda} x \cdot \cos \sqrt{\lambda}(1-x)+O\left(|\lambda|^{-1 / 2} e^{|\operatorname{Im} \sqrt{\lambda}|}\right) \\
& =\cos \sqrt{\lambda} \cdot\left(1+O\left(|\lambda|^{-1 / 2}\right)\right.
\end{aligned}
$$

and

$$
F_{2}(x, \lambda)=O\left(|\lambda|^{-1 / 2} e^{\mid \operatorname{Im} \sqrt{\lambda \mid}}\right)
$$

Thus,

$$
f_{1}(x, \lambda)=1+O\left(|\lambda|^{-1 / 2}\right) \quad \text { and } \quad f_{2}(x, \lambda)=O\left(|\lambda|^{-1 / 2}\right) \quad \text { as } \quad|\lambda|=\pi^{2} m^{2} \rightarrow \infty .
$$

Since $f_{1}$ and $f_{2}$ are entire with respect to $\lambda$, the maximum principle implies that $f_{1}(x, \lambda) \equiv 1$ and $f_{2}(x, \lambda) \equiv 0$ for all $x \in[0,1]$ and $\lambda \in \mathbb{C}$. In other words,

$$
\begin{aligned}
& (\varphi(x, \lambda, p)-\varphi(x, \lambda, q)) \xi_{b}^{\prime}(x, \lambda, q)-\left(\xi_{b}(x, \lambda, p)-\xi_{b}(x, \lambda, q)\right) \varphi^{\prime}(x, \lambda, q)=0, \\
& (\varphi(x, \lambda, p)-\varphi(x, \lambda, q)) \xi_{b}(x, \lambda, q)-\left(\xi_{b}(x, \lambda, p)-\xi_{b}(x, \lambda, q)\right) \varphi(x, \lambda, q)=0 .
\end{aligned}
$$

This gives $\varphi(x, \lambda, p)=\varphi(x, \lambda, q)$ for all $x \in[0,1]$ and $\lambda \neq \lambda_{n}(q), n \geq 0$. Thus, $p=q$. 


\section{§3. AnAlyticity, ASYMPTOTICS, AND LOCAL ISOMORPHISM}

Lemma 3.1. Suppose $(q ; b) \in L_{\mathbb{C}}^{2}(0,1) \times \mathbb{C}$ and $N>2(\|q\|+|b|) e^{\|q\|}$. Then, counting with multiplicities, $w(\lambda)$ has exactly $N$ roots in the disk $\left\{\lambda:|\lambda|<\pi^{2} N^{2}\right\}$ and for each $n>N$ exactly one simple root $\lambda_{n}(q, b)$ in the region $\left\{\lambda:\left|\sqrt{\lambda}-k_{n}^{0}\right|<\pi / 4\right\}$. There are no other roots. Moreover, the following rough asymptotics is valid:

$$
\lambda_{n}(q, b)=\lambda_{n}^{0}+O(1) \quad \text { as } \quad n \rightarrow \infty,
$$

uniformly on bounded subsets of $L_{\mathbb{C}}^{2}(0,1) \times \mathbb{C}$.

Proof. The standard estimates of $\varphi$ and $\varphi^{\prime}$ (see [PT87, p. 13]) give

$$
\left|\varphi^{\prime}(1, \lambda, q)-\cos \sqrt{\lambda}\right| \leq|\lambda|^{-\frac{1}{2}} \cdot\|q\| e^{\|q\|+|\operatorname{Im} \sqrt{\lambda}|}, \quad|\varphi(1, \lambda, q)| \leq|\lambda|^{-\frac{1}{2}} \cdot e^{\|q\|+|\operatorname{Im} \sqrt{\lambda}|} .
$$

Hence,

$$
|w(\lambda, q, b)-\cos \sqrt{\lambda}| \leq|\lambda|^{-\frac{1}{2}} \cdot(\|q\|+|b|) e^{|| q \|+|\operatorname{Im} \sqrt{\lambda}|}<\frac{1}{2} N|\lambda|^{-\frac{1}{2}} e^{|\operatorname{Im} \sqrt{\lambda}|} .
$$

Let $\lambda \in L_{N} \cup \bigcup_{n>N}\left(L_{n} \cup l_{n}\right)$, where $L_{n}=\left\{\lambda:|\lambda|=\pi^{2} n^{2}\right\}$ and $l_{n}=\left\{\lambda:\left|\sqrt{\lambda}-k_{n}^{0}\right|=\right.$ $\pi / 4\}$. Using the simple estimate $4|\cos \sqrt{\lambda}|>e^{|\operatorname{Im} \sqrt{\lambda}|}$ on these contours, we obtain

$$
|w(\lambda, q, b)-\cos \sqrt{\lambda}|<2 N|\lambda|^{-1 / 2} \cdot|\cos \sqrt{\lambda}|<|\cos \sqrt{\lambda}|, \quad \lambda \in L_{N} \cup \bigcup_{n>N}\left(L_{n} \cup l_{n}\right) .
$$

Therefore, by the Rouché theorem, in each region bounded by these contours, $w(\cdot, q, b)$ has as many roots as $\cos \sqrt{\lambda}$ has. Furthermore, formulas (2.4), (2.5) show that $0=$ $w\left(\lambda_{n}, q, b\right)=\cos \sqrt{\lambda_{n}}+O\left(n^{-1}\right)$ as $n \rightarrow \infty$, uniformly on bounded subsets of $L_{\mathbb{C}}^{2}(0,1) \times \mathbb{C}$. Since $k_{n}^{0}$ is a simple root of the function $\cos z$, we have $\sqrt{\lambda_{n}}=k_{n}^{0}+O\left(n^{-1}\right)$, which is equivalent to (3.1).

We introduce a scalar product in $L_{\mathbb{C}}^{2}(0,1) \times \mathbb{C}$ by

$$
\langle(q ; b),(p ; h)\rangle=\int_{0}^{1} q(t) \overline{p(t)} d t+b \bar{h} .
$$

In what follows, we write $d_{(q ; b)} \sigma(q, b)=(f ; g)$ if and only if

$$
\left[d_{(q ; b)} \sigma(q, b)\right](p ; h)=\langle(p ; h), \overline{(f ; g)}\rangle \text { for all }(p ; h) \in L^{2}(0,1) \times \mathbb{R} .
$$

We use the similar symbols $\partial_{q}, \partial_{b}$ for the corresponding partial derivatives.

Lemma 3.2. (i) All $\lambda_{n}(q, b), \mu_{n}(q, b)$, and $\nu_{n}(q, b), n \geq 0$, are real-analytic on $L^{2}(0,1) \times$ $\mathbb{R}$. Their derivatives are given by

$$
\begin{aligned}
& d_{(q(x) ; b)} \lambda_{n}(q, b)=\left(\psi_{n}^{2}(x) ; \psi_{n}^{2}(1)\right), \quad d_{(q(x) ; b)} \mu_{n}(q, b)=\left(\psi_{n}^{2}(x)-1 ; \psi_{n}^{2}(1)-2\right), \\
& d_{(q(x) ; b)} \nu_{n}(q, b)=\left(\left(\psi_{n} \chi_{n}\right)(x) ;\left(\psi_{n} \chi_{n}\right)(1)\right),
\end{aligned}
$$

where $\psi_{n}(x)=\psi_{n}(x, q, b)$ is the $n$th normalized eigenfunction of the operator $H$, and

$$
\chi_{n}(x)=\chi_{n}(x, q, b):=\frac{\vartheta\left(x, \lambda_{n}, q\right)}{\psi_{n}^{\prime}(0, q, b)}-\psi_{n}(x, q, b) \int_{0}^{1}(\varphi \vartheta)\left(t, \lambda_{n}, q\right) d t .
$$

(ii) Let $(q ; b) \in L^{2}(0,1) \times \mathbb{R}$. Then each of the functions $\lambda_{n}, \mu_{n}$, and $\nu_{n}, n \geq 0$, admits analytic continuation from $L^{2}(0,1) \times \mathbb{R}$ to some complex ball

$$
\left\{(p ; h) \in L_{\mathbb{C}}^{2}(0,1) \times \mathbb{C}:\|(p-q ; b-h)\|<\varepsilon\right\},
$$

where the radius $\varepsilon=\varepsilon(q, b)>0$ does not depend on $n$.

Remark 3.3. Since $\psi_{n}(0)=0$, we have $\psi_{n}(x) \equiv \psi_{n}^{\prime}(0) \varphi(x), x \in[0,1]$. Therefore, $\chi_{n}(x)$ solves (2.1) for $\lambda=\lambda_{n}$, and $\left\{\chi_{n}, \psi_{n}\right\}=1, \int_{0}^{1} \psi_{n}(x) \chi_{n}(x) d x=0$. Observe that these conditions determine $\chi_{n}$ uniquely. 
Proof of Lemma 3.2. (i) It is well known (see [PT87, p. 21]) that

$$
\begin{aligned}
\partial_{q(x)} \varphi(1, \lambda, q) & =\varphi(x)(\vartheta(x) \varphi(1)-\varphi(x) \vartheta(1)), \\
\partial_{q(x)} \varphi^{\prime}(1, \lambda, q) & =\varphi(x)\left(\vartheta(x) \varphi^{\prime}(1)-\varphi(x) \vartheta^{\prime}(1)\right),
\end{aligned}
$$

where $\varphi(x)=\varphi(x, \lambda, q), \vartheta(x)=\vartheta(x, \lambda, q)$, and so on. This gives

$$
d_{(q(x) ; b)} w(\lambda, q, b)=\left(\left(\left(\varphi^{\prime}+b \varphi\right)(1) \vartheta(x)-\left(\vartheta^{\prime}+b \vartheta\right)(1) \varphi(x)\right) \varphi(x) ; \varphi(1)\right) .
$$

Note that

$$
\left(\varphi^{\prime}+b \varphi\right)(1) \vartheta(x)-\left(\vartheta^{\prime}+b \vartheta\right)(1) \varphi(x) \equiv-\xi_{b}(x), \quad x \in[0,1],
$$

because these two functions are solutions of (2.1) with the same initial data at $x=1$. Therefore,

$$
d_{(q(x) ; b)} w(\lambda, q, b)=\left(-\varphi(x) \xi_{b}(x) ;-\varphi(1) \xi_{b}(1)\right) .
$$

Recall that the function $w(\cdot, \cdot, \cdot)$ is entire and $w\left(\lambda_{n}(q, b), q, b\right)=0$. Then, by the implicit function theorem and Lemma 2.1

$$
d_{(q(x) ; b)} \lambda_{n}(q, b)=-\frac{d_{(q(x) ; b)} w\left(\lambda_{n}, q, b\right)}{\dot{w}\left(\lambda_{n}, q, b\right)}=\left(\psi_{n}^{2}(x) ; \psi_{n}^{2}(1)\right) .
$$

Since $\mu_{n}(q, b)=\lambda_{n}(q, b)-\lambda_{n}^{0}-\int_{0}^{1} q(t) d t-2 b$, we get

$$
d_{(q(x) ; b)} \mu_{n}(q, b)=d_{(q(x) ; b)} \lambda_{n}(q, b)-(1 ; 2)=\left(\psi_{n}^{2}(x)-1 ; \psi_{n}^{2}(1)-2\right) .
$$

Furthermore, the definition (1.3) implies that

$$
d_{(q(x) ; b)} \nu_{n}(q, b)=\frac{d_{(q(x) ; b)} \varphi\left(1, \lambda_{n}(q, b), q\right)}{\varphi(1)}=\frac{\left(\partial_{q(x)} \varphi\left(1, \lambda_{n}, q\right) ; 0\right)+\dot{\varphi}(1) d_{(q(x) ; b)} \lambda_{n}(q, b)}{\varphi(1)} .
$$

Substituting $\partial_{q(x)} \varphi\left(1, \lambda_{n}, q\right)$ and $d_{(q(x) ; b)} \lambda_{n}(q, b)$ in this formula and using the identity $\psi_{n}(x) \equiv \psi_{n}^{\prime}(0) \varphi(x), x \in[0,1]$, we arrive at the relation

$$
d_{(q(x) ; b)} \nu_{n}(q, b)=\left(\psi_{n}(x) \chi_{n}(x) ; \psi_{n}(1) \chi_{n}(1)\right), \quad \chi_{n}(x) \equiv \frac{\vartheta\left(x, \lambda_{n}, q\right)}{\psi_{n}^{\prime}(0, q, b)}-C_{n} \psi_{n}(x, q, b),
$$

where $C_{n}=C_{n}(q, b)$ is some constant. In order to find $C_{n}$, observe that $\nu_{n}(q+c, b)=$ $\nu_{n}(q, b)$ for all $c \in \mathbb{R}$. Therefore,

$$
0=\left\langle\partial_{q(x)} \nu_{n}(q, b), 1\right\rangle=\int_{0}^{1} \psi_{n}(x) \chi_{n}(x) d x=\int_{0}^{1} \varphi(x) \vartheta(x) d x-C_{n} \int_{0}^{1} \psi_{n}^{2}(x) d x .
$$

Since $\int_{0}^{1} \psi_{n}^{2}(x) d x=1$, this gives $C_{n}=\int_{0}^{1} \varphi(x) \vartheta(x) d x$. (ii) The proof repeats PT87, p. 51] for eigenvalues and [PT87, p. 64] for norming constants.

Lemma 3.4. (i) The following asymptotic formulas hold:

$$
\begin{array}{cl}
\psi_{n}(x, q, b)=\sqrt{2} \sin k_{n}^{0} x+O\left(n^{-1}\right), & \chi_{n}(x, q, b)=\frac{\cos k_{n}^{0} x}{\sqrt{2} k_{n}^{0}}+O\left(n^{-2}\right), \quad n \rightarrow \infty, \\
\mu_{n}(q, b)=-\widehat{q}_{n+\frac{1}{2}}^{(\cos )}+O\left(n^{-1}\right), \quad \nu_{n}(q, b)=\nu_{n}^{0}+\frac{\widehat{q}_{n+\frac{1}{2}}^{(\sin )}}{2 k_{n}^{0}}+O\left(n^{-2}\right), \quad n \rightarrow \infty,
\end{array}
$$

uniformly on bounded subsets of $[0,1] \times L_{\mathbb{C}}^{2}(0,1) \times \mathbb{C}$, where

$$
\widehat{q}_{n+\frac{1}{2}}^{(\cos )}=\int_{0}^{1} q(x) \cos 2 k_{n}^{0} x d x, \quad \widehat{q}_{n+\frac{1}{2}}^{(\sin )}=\int_{0}^{1} q(x) \sin 2 k_{n}^{0} x d x .
$$

(ii) The mapping $\Phi$ is real-analytic on $L^{2}(0,1) \times \mathbb{R}$, and its Fréchet derivative is given by

$$
\left[d_{(q ; b)} \Phi\right](\cdot)=\left(\langle\cdot,(1 ; 2)\rangle ;\left\{\left\langle\cdot, X_{n}\right\rangle\right\}_{n=0}^{+\infty} ;\left\{\left\langle\cdot, Y_{n}\right\rangle\right\}_{n=0}^{+\infty}\right),
$$


where

$$
X_{n}=\left(\psi_{n}^{2}(x)-1 ; \psi_{n}^{2}(1)-2\right), \quad Y_{n}=\left(\left(\psi_{n} \chi_{n}\right)(x) ;\left(\psi_{n} \chi_{n}\right)(1)\right), \quad n \geq 0 .
$$

Proof. (i) Let $\lambda_{n}=\lambda_{n}(q, b)$. The asymptotics (3.1) shows that $\sqrt{\lambda_{n}}=k_{n}^{0}+O\left(n^{-1}\right)$. Formulas (2.2)-(2.5) imply that

$$
\varphi\left(x, \lambda_{n}, q\right)=\frac{\sin k_{n}^{0} x}{k_{n}^{0}}+O\left(n^{-2}\right), \quad\left\|\varphi\left(\cdot, \lambda_{n}, q\right)\right\|^{2}=\int_{0}^{1} \varphi^{2}\left(x, \lambda_{n}, q\right) d x=\frac{1}{2 \lambda_{n}^{0}}+O\left(n^{-3}\right) .
$$

Hence,

$$
\psi_{n}(x, q, b)=\frac{\varphi\left(x, \lambda_{n}, q\right)}{\left\|\varphi\left(\cdot, \lambda_{n}, q\right)\right\|}=\sqrt{2} \sin k_{n}^{0} x+O\left(n^{-1}\right) .
$$

Thus, $\psi_{n}^{\prime}(0, q, b)=\left\|\varphi\left(\cdot, \lambda_{n}, q\right)\right\|^{-1}=\sqrt{2} k_{n}^{0}+O(1)$. Also,

$$
\vartheta\left(x, \lambda_{n}, q\right)=\cos k_{n}^{0} x+O\left(n^{-1}\right), \quad \int_{0}^{1}(\vartheta \varphi)\left(x, \lambda_{n}, q\right) d x=O\left(n^{-2}\right) .
$$

Using (3.4), we obtain the second asymptotics in (3.5). Finally, Lemma 3.2 and (3.5) imply that

$$
\begin{aligned}
\lambda_{n}(q, b) & =\int_{0}^{1}\left[d_{(q ; b)} \mu_{n}(t q, t b)\right](q ; b) d t=\lambda_{n}^{0}+\left\langle\left(2 \sin ^{2} k_{n}^{0} x-1 ; 0\right),(q(x) ; b)\right\rangle+O\left(n^{-1}\right), \\
\nu_{n}(q, b) & =\nu_{n}^{0}+\int_{0}^{1}\left[d_{(q ; b)} \nu_{n}(t q, t b)\right](q ; b) d t \\
& =\nu_{n}^{0}+\frac{\left\langle\left(\sin k_{n}^{0} x \cos k_{n}^{0} x ; 0\right),(q(x) ; b)\right\rangle}{k_{n}^{0}}+O\left(n^{-2}\right) .
\end{aligned}
$$

(ii) As has been shown above, the mapping $\Phi: L_{\mathbb{C}}^{2}(0,1) \times \mathbb{C} \rightarrow \mathbb{C} \times \ell^{2} \times \ell_{1}^{2}$ is locally bounded near each real point $(q ; b) \in L^{2}(0,1) \times \mathbb{R}$, and all the "coordinate functions" $\int_{0}^{1} q(t) d t+2 b, \lambda_{n}(q, b), \nu_{n}(q, b)-\nu_{n}^{0}$ are (complex-)analytic (see Lemma $3.2(\mathrm{ii})$ ). Therefore (see, e.g., [PT87, p. 138]), $\Phi$ is a real-analytic mapping and its Fréchet derivative is given by (3.7).

Lemma 3.5. Let $(q ; b) \in L^{2}(0,1) \times \mathbb{R}$, and let

$$
\begin{array}{lll}
X_{n}=\left(\psi_{n}^{2}(x)-1 ; \psi_{n}^{2}(1)-2\right), & Y_{n}=\left(\left(\psi_{n} \chi_{n}\right)(x) ;\left(\psi_{n} \chi_{n}\right)(1)\right), & n \geq 0, \\
Z_{m}=\left(-2\left(\psi_{m} \chi_{m}\right)^{\prime}(x) ;\left(\psi_{m} \chi_{m}\right)(1)\right), & T_{m}=\left(2\left(\psi_{m}^{2}\right)^{\prime}(x) ;-\psi_{m}^{2}(1)\right), & m \geq 0 .
\end{array}
$$

Then the system $(1 ; 0),\left\{Z_{m}\right\}_{m=0}^{+\infty},\left\{T_{m}\right\}_{m=0}^{+\infty}$ forms the basis biorthogonal to the system $(1 ; 2),\left\{X_{n}\right\}_{n=0}^{+\infty},\left\{Y_{n}\right\}_{n=0}^{+\infty}$. In other words,

$$
\begin{aligned}
\langle(1 ; 0),(1 ; 2)\rangle & =1, & \left.\left\langle Z_{m} 1 ; 2\right)\right\rangle & =0, & \left\langle T_{m}(1 ; 2)\right\rangle & =0, \\
\left\langle(1 ; 0) X_{n}\right\rangle & =0, & \left\langle Z_{m} X_{n}\right\rangle & =\delta_{m n}, & \left\langle T_{m} X_{n}\right\rangle & =0, \\
\left\langle(1 ; 0) Y_{n}\right\rangle & =0, & \left\langle Z_{m} Y_{n}\right\rangle & =0, & \left\langle T_{m} Y_{n}\right\rangle & =\delta_{m n},
\end{aligned}
$$

Proof. By definition, we have $\langle(1 ; 0),(1 ; 2)\rangle=1$, and $\left\langle Z_{m},(1 ; 2)\right\rangle=\left\langle T_{m},(1 ; 2)\right\rangle=0$ because $\psi_{m}(0)=0$. Since $\int_{0}^{1} \psi_{n}^{2}(x) d x=1$ and $\int_{0}^{1}\left(\psi_{n} \chi_{n}\right)(x) d x=0$ (see Remark 3.3), we obtain

$$
\left\langle(1 ; 0), X_{n}\right\rangle=\left\langle(1 ; 0), Y_{n}\right\rangle=0 .
$$


Next, integration by parts gives

$$
\begin{aligned}
\left\langle Z_{m}, X_{n}\right\rangle & =-2 \int_{0}^{1}\left(\psi_{n}^{2}(x)-1\right)\left(\psi_{m} \chi_{m}\right)^{\prime}(x) d x+\left(\psi_{n}^{2}(1)-2\right)\left(\psi_{m} \chi_{m}\right)(1) \\
& =-2 \int_{0}^{1} \psi_{n}^{2}(x)\left(\psi_{m} \chi_{m}\right)^{\prime}(x) d x+\left(\psi_{n}^{2}\right)(1)\left(\psi_{m} \chi_{m}\right)(1) \\
& =-\int_{0}^{1}\left(\psi_{n}^{2}\left(\psi_{m} \chi_{m}\right)^{\prime}-\left(\psi_{n}^{2}\right)^{\prime} \psi_{m} \chi_{m}\right)(x) d x \\
& =-\int_{0}^{1}\left(\left\{\psi_{n}, \psi_{m}\right\} \cdot \psi_{n} \chi_{m}+\psi_{n} \psi_{m} \cdot\left\{\psi_{n}, \chi_{m}\right\}\right)(x) d x
\end{aligned}
$$

If $n \neq m$, then $\left\{\psi_{n}, \psi_{m}\right\}^{\prime}=\left(\lambda_{n}-\lambda_{m}\right) \psi_{n} \psi_{m},\left\{\psi_{n}, \chi_{m}\right\}^{\prime}=\left(\lambda_{n}-\lambda_{m}\right) \psi_{n} \chi_{m}$. Hence,

$$
\left\langle Z_{m}, X_{n}\right\rangle=-\left.\frac{\left\{\psi_{n}, \psi_{m}\right\}\left\{\psi_{n}, \chi_{m}\right\}}{\lambda_{n}-\lambda_{m}}\right|_{0} ^{1}=0, \quad n \neq m,
$$

because $\left\{\psi_{n}, \psi_{m}\right\}(0)=\left\{\psi_{n}, \psi_{m}\right\}(1)=0$. If $n=m$, then $\left\{\psi_{n}, \psi_{m}\right\}=0,\left\{\psi_{n}, \chi_{m}\right\}=-1$ and

$$
\left\langle Z_{n}, X_{n}\right\rangle=\int_{0}^{1} \psi_{n}^{2}(x) d x=1, \quad n \geq 0 .
$$

The proof of the remaining identities is similar.

Theorem 3.6 (Local isomorphism). For each $(q ; b) \in L^{2}(0,1) \times \mathbb{R}$, the linear operator $d_{(q ; b)} \Phi: L^{2}(0,1) \times \mathbb{R} \rightarrow \mathbb{R} \times \ell^{2} \times \ell_{1}^{2}$ given by (3.7) is invertible.

Proof. By Lemma 3.4 (i),

$X_{n}=(-\cos \pi(2 n+1) x ; 0)+O\left(n^{-1}\right), \quad 2 k_{n}^{0} Y_{n}=(\sin \pi(2 n+1) x ; 0)+O\left(n^{-1}\right), \quad n \geq 0$.

Note that the vectors

$$
(0 ; 1) ;\{(\sqrt{2} \cos \pi(2 n+1) x ; 0)\}_{n=0}^{+\infty} ;\{(\sqrt{2} \sin \pi(2 n+1) x ; 0)\}_{n=0}^{+\infty}
$$

form an orthonormal basis in $L^{2}(0,1) \times \mathbb{R}$, and that the error terms are square integrable. Consequently, $d_{(q(x) ; b)} \Phi$ is a Fredholm operator. By Lemma 3.5, the vectors $(1 ; 2),\left\{X_{n}\right\}_{n=0}^{+\infty},\left\{Y_{n}\right\}_{n=0}^{+\infty}$ are linearly independent. Using standard functional analysis arguments (see, e.g., [PT87, p. 163]), we deduce that $\left(d_{(q ; b)} \Phi\right)^{-1}$ is bounded.

\section{§4. Proof of Theorem 1.1 and Corollaries 1.3 , 1.2}

Lemma 4.1. For each data $\left(c^{*} ;\left\{\mu_{n}^{*}\right\}_{n=0}^{+\infty}\right) \in \mathbb{R} \times \mathcal{M}$, there exists a potential $q^{*} \in L^{2}(0,1)$ such that $\lambda_{n}\left(q^{*}, 0\right)=\lambda_{n}^{0}+c^{*}+\mu_{n}^{*}$ for all $n \geq 0$.

Proof. Set

$$
\lambda_{n+\frac{1}{2}}^{*}=\frac{n+\frac{5}{4}}{2(n+1)} \cdot \lambda_{n}^{*}+\frac{n+\frac{3}{4}}{2(n+1)} \cdot \lambda_{n+1}^{*}, \quad n \geq 0 .
$$

Then, $\lambda_{0}^{*}<\lambda_{1 / 2}^{*}<\lambda_{1}^{*}<\lambda_{3 / 2}^{*}<\cdots$ and $\lambda_{m / 2}^{*}=\pi^{2}(m / 2)^{2}+c^{*}+\mu_{m / 2}^{*}$, where $\left\{\mu_{m / 2}^{*}\right\}_{m=0}^{+\infty} \in \ell^{2}$. Thus (see, e.g., [PT87]), there exists an "even" potential $q \in L^{2}(0,2)$, $q(x) \equiv q(2-x), x \in[0,2]$, such that $\left\{\lambda_{m / 2}^{*}\right\}_{m=0}^{+\infty}$ is the Dirichlet spectrum of $q$ on $[0,2]$. Put $q^{*}=\left.q\right|_{[0,1]}$. Then $\lambda_{n}\left(q^{*}, 0\right)=\lambda_{2 n / 2}^{*}=\lambda_{n}^{*}$ for all $n \geq 0$. 
Lemma 4.2. Let $(q ; b) \in L^{2}(0,1) \times \mathbb{R}, n \geq 0$, and $t \in \mathbb{R}$. Denote

$$
\begin{aligned}
q_{n}^{t}(x) & :=q(x)-2 \frac{d^{2}}{d x^{2}} \log \eta_{n}^{t}(x, q, b), \\
\eta_{n}^{t}(x, q, b) & :=1+\left(e^{t}-1\right) \int_{x}^{1} \psi_{n}^{2}(t, q, b) d t, \quad x \in[0,1], \\
b_{n}^{t} & :=b-\left(e^{t}-1\right) \psi_{n}^{2}(1, q, b) .
\end{aligned}
$$

Then $\left(q_{n}^{t} ; b_{n}^{t}\right) \in L^{2}(0,1) \times \mathbb{R}$ and

$$
\lambda_{m}\left(q_{n}^{t}, b_{n}^{t}\right)=\lambda_{m}(q, b), \quad \nu_{m}\left(q_{n}^{t}, b_{n}^{t}\right)=\nu_{m}(q, b)+t \delta_{m n}
$$

for all $m \geq 0$.

Proof. Let $\psi_{n}(x)=\psi_{n}(x, q, b)$, and so on. Repeating the arguments of PT87, pp. 91-93] or using direct calculations, it is easy to check that for all $m \geq 0$ the functions

$$
\widetilde{\psi}_{m}(x)=\psi_{m}(x)-\left(e^{t}-1\right) \frac{\psi_{n}(x)}{\eta_{n}^{t}(x)} \int_{x}^{1} \psi_{n}(t) \psi_{m}(t) d t
$$

(in particular, $\widetilde{\psi}_{n}(x)=\psi_{n}(x) / \eta_{n}^{t}(x)$ ) solve the equation $-\psi^{\prime \prime}+q_{n}^{t}(x) \psi=\lambda_{m}(q, b) \psi$. Since $\psi_{m}(0)=\psi_{n}(0)=0$, we have $\widetilde{\psi}_{m}(0)=0$. The crucial point is the new boundary condition at $x=1$. Observe that

$$
\widetilde{\psi}_{m}^{\prime}(1)+b_{n}^{t} \widetilde{\psi}_{m}(1)=\psi_{m}^{\prime}(1)+\left(e^{t}-1\right) \psi_{n}^{2}(1) \psi_{m}(1)+b_{n}^{t} \widetilde{\psi}_{m}(1)=\psi_{m}^{\prime}(1)+b \psi_{m}(1)=0
$$

for all $m \geq 0$. Therefore, $\lambda_{m}\left(q_{n}^{t}, b_{n}^{t}\right)=\lambda_{m}(q, b), m \geq 0$ (there are no other roots; see Lemma 3.1). Furthermore,

$$
\widetilde{\psi}_{m}^{\prime}(0)=\psi_{m}^{\prime}(0), \quad m \neq n, \quad \text { and } \quad \widetilde{\psi}_{n}^{\prime}(0)=e^{-t} \widetilde{\psi}_{n}^{\prime}(0) .
$$

By the definition (1.3), this gives $\nu_{m}\left(q_{n}^{t}, b_{n}^{t}\right)=\nu_{m}(q, b)+t \delta_{m n}$ for all $m \geq 0$.

Proof of Theorem 1.1. (ii) See Lemma 2.2 .

(i) Lemma 3.4 shows that $\Phi$ maps $L^{2}(0,1) \times \mathbb{R}$ into $\mathbb{R} \times \mathcal{M} \times \ell_{1}^{2}$; $\Phi$ is real-analytic and its Fréchet derivative is given by (3.7). By Theorem 3.6, $\Phi$ is a local isomorphism. Furthermore, by Theorem [2.3, $\Phi$ is one-to-one. Thus, it suffices to prove that $\Phi$ is onto.

Let $c^{*} \in \mathbb{R},\left\{\mu_{n}^{*}\right\}_{n=0}^{+\infty} \in \mathcal{M}$, and $\left\{\eta_{n}^{*}\right\}_{n=0}^{+\infty} \in \ell_{1}^{2}$ be arbitrary data. By Lemma 4.1 there exists a potential $q^{*} \in L^{2}(0,1)$ such that $\lambda_{n}\left(q^{*}, 0\right)=\lambda_{n}^{*}=\lambda_{n}^{0}+c^{*}+\mu_{n}^{*}$ for all $n \geq 0$. Let $\eta_{n}=\nu_{n}\left(q^{*}, 0\right)-\nu_{n}^{0}, n \geq 0$. Note that

$$
\left\|\left(\eta_{0}, \ldots, \eta_{N-1}, \eta_{N}^{*}, \eta_{N+1}^{*}, \ldots\right)-\left(\eta_{0}, \ldots, \eta_{N-1}, \eta_{N}, \ldots\right)\right\|_{\ell_{1}^{2}} \rightarrow 0 \text { as } N \rightarrow \infty .
$$

By Theorem 3.6 the mapping $\Phi$ is invertible in some neighborhood of the point $\Phi\left(q^{*}, 0\right)=$ $\left(c^{*} ;\left\{\mu_{n}^{*}\right\}_{n=0}^{+\infty} ;\left\{\eta_{n}\right\}_{n=0}^{+\infty}\right) \in \mathbb{R} \times \mathcal{M} \times \ell_{1}^{2}$. Hence, for some (sufficiently large) integer $N$ and some $\left(q^{(N)} ; b^{(N)}\right) \in L^{2}(0,1) \times \mathbb{R}$, we have

$$
\left(c^{*} ;\left\{\mu_{n}^{*}\right\}_{n=0}^{+\infty} ;\left(\eta_{0}, \ldots, \eta_{N-1}, \eta_{N}^{*}, \eta_{N+1}^{*}, \ldots\right)\right)=\Phi\left(q^{(N)}, b^{(N)}\right) .
$$

Applying Lemma 4.2 step-by-step, we construct a sequence of potentials and boundary constants

$\left(q^{(k)} ; b^{(k)}\right)=\left(q^{(k+1)} ; b^{(k+1)}\right)_{k}^{t_{k}} \in L^{2}(0,1) \times \mathbb{R}, \quad t_{k}=\eta_{k}^{*}-\eta_{k}, \quad k=N-1, N-2, \ldots, 0$, such that

$$
\Phi\left(q^{(k)}, b^{(k)}\right)=\left(c^{*} ;\left\{\mu_{n}^{*}\right\}_{n=0}^{+\infty} ;\left(\eta_{0}, \ldots, \eta_{k-1}, \eta_{k}^{*}, \eta_{k+1}^{*}, \ldots\right)\right) .
$$

In particular, $\lambda_{n}\left(q^{(0)}, b^{(0)}\right)=\lambda_{n}^{*}$ and $\nu_{n}\left(q^{(0)}, b^{(0)}\right)-\nu_{n}^{0}=\eta_{n}^{*}$ for all $n \geq 0$. 
Proof of Corollary 1.3. Suppose $b, c^{*} \in \mathbb{R},\left\{\mu_{n}^{*}\right\}_{n=0}^{+\infty} \in \mathcal{M}, m \geq 0$, and

$$
\mathcal{N}^{b}=\left\{\left\{\nu_{n}-\nu_{n}^{0}\right\}_{n=0}^{+\infty} \in \ell_{1}^{2}: \sum_{n=0}^{+\infty}\left(2-\frac{e^{\nu_{n}}}{\left|\dot{w}^{*}\left(\lambda_{n}^{*}\right)\right|}\right)=b\right\} .
$$

By Theorem 1.1, the mapping $q \mapsto\left\{\nu_{n}(q, b)-\nu_{n}^{0}\right\}_{n=0}^{+\infty}$ is a bijection between $\operatorname{Iso}_{b}\left[\left\{\lambda_{n}^{*}\right\}_{n=0}^{+\infty}\right]$ and $\mathcal{N}^{b}$. We introduce the projection

$$
P_{m}:\left\{\nu_{n}-\nu_{n}^{0}\right\}_{n=0}^{+\infty} \mapsto\left\{\nu_{n}-\nu_{n}^{0}\right\}_{n=0, n \neq m}^{+\infty} .
$$

It is easily seen that

$$
P_{m}: \mathcal{N}^{b} \rightarrow \mathcal{N}_{m}^{b}=\left\{\left\{\nu_{n}-\nu_{n}^{0}\right\}_{n=0, n \neq m}^{+\infty} \in \ell_{1}^{2}: \sum_{n=0, n \neq m}^{+\infty}\left(2-\frac{e^{\nu_{n}}}{\left|\dot{w}^{*}\left(\lambda_{n}^{*}\right)\right|}\right)>b-2\right\}
$$

is a bijection. Moreover, $\partial b / \partial \nu_{m}=-e^{\nu_{m}}\left|\dot{w}^{*}\left(\lambda_{m}\right)\right|^{-1} \neq 0$. Thus, from the implicit function theorem it follows that $\mathcal{N}^{b} \subset \ell_{1}^{2}$ is a real-analytic manifold and $P_{m}: \mathcal{N}^{b} \rightarrow \mathcal{N}_{m}^{b}$ is a real-analytic isomorphism. Using Theorem 1.1, we conclude that

$$
\mathrm{Iso}_{b}\left[\left\{\lambda_{n}^{*}\right\}_{n=0}^{+\infty}\right] \times\{b\}=\Phi^{-1}\left(\left\{c^{*}\right\} \times\left\{\left\{\mu_{n}^{*}\right\}_{n=0}^{+\infty}\right\} \times \mathcal{N}^{b}\right)
$$

is a real-analytic manifold and the mapping

$$
q \mapsto\left\{\nu_{n}(q, b)-\nu_{n}^{0}\right\}_{n=0}^{+\infty}, \quad \operatorname{Iso}_{b}\left[\left\{\lambda_{n}^{*}\right\}_{n=0}^{+\infty}\right] \rightarrow \mathcal{N}^{b},
$$

is a real-analytic isomorphism. Therefore, the mapping $q \mapsto\left\{\nu_{n}(q, b)-\nu_{n}^{0}\right\}_{n=0, n \neq m}^{+\infty}$ is the composition of two real-analytic isomorphisms.

For each $\left\{\lambda_{n}\right\}_{n=0}^{+\infty}$ such that $\lambda_{n}=\lambda_{n}^{0}+c+\mu_{n}, c \in \mathbb{R},\left\{\mu_{n}\right\}_{n=0}^{+\infty} \in \mathcal{M}$, we introduce the function

$$
W(\lambda)=W\left(\lambda ; \lambda_{0}, \lambda_{1}, \ldots\right):=\cos \sqrt{\lambda} \cdot \prod_{k=0}^{+\infty} \frac{\lambda-\lambda_{k}}{\lambda-\lambda_{k}^{0}}, \quad \lambda \in \mathbb{C} .
$$

Lemma 4.3. Let $c \in \mathbb{R},\left\{\mu_{n}\right\}_{n=0}^{+\infty} \in \mathcal{M}$ and $n \geq 0$. Then

$$
\frac{\partial}{\partial \lambda_{0}}\left|\dot{W}\left(\lambda_{n}\right)\right|^{-1}>0, \quad \lim _{\lambda_{0} \downarrow-\infty}\left|\dot{W}\left(\lambda_{n}\right)\right|^{-1}=0 .
$$

Furthermore,

$$
\lim _{\lambda_{0} \uparrow \lambda_{1}}\left|\dot{W}\left(\lambda_{0}\right)\right|^{-1}=\lim _{\lambda_{0} \uparrow \lambda_{1}}\left|\dot{W}\left(\lambda_{1}\right)\right|^{-1}=+\infty
$$

Proof. Denote

$$
W_{\beta}(\lambda):=W\left(\lambda ; \lambda_{0}+\beta, \lambda_{1}, \lambda_{2}, \ldots\right) \equiv \frac{\lambda-\lambda_{0}-\beta}{\lambda-\lambda_{0}} \cdot W(\lambda) \equiv\left(1-\frac{\beta}{\lambda-\lambda_{0}}\right) W(\lambda), \quad \lambda \in \mathbb{C} .
$$

Let $n \geq 1$. Since $W\left(\lambda_{n}\right)=0$ and $\dot{W}\left(\lambda_{n}\right) \neq 0$, we have

$$
\dot{W}_{\beta}\left(\lambda_{n}\right)=\left(1-\frac{\beta}{\lambda_{n}-\lambda_{0}}\right) \dot{W}\left(\lambda_{n}\right), \quad \frac{\partial \dot{W}\left(\lambda_{n}\right)}{\partial \lambda_{0}}=\left.\frac{\partial}{\partial \beta} \dot{W}_{\beta}\left(\lambda_{n}\right)\right|_{\beta=0}=-\frac{\dot{W}\left(\lambda_{n}\right)}{\lambda_{n}-\lambda_{0}} .
$$

Hence,

and

$$
\frac{\partial}{\partial \lambda_{0}}\left|\dot{W}\left(\lambda_{n}\right)\right|^{-1}=\left|\dot{W}\left(\lambda_{n}\right)\right|^{-2} \cdot \frac{\left|\dot{W}\left(\lambda_{n}\right)\right|}{\lambda_{n}-\lambda_{0}}>0
$$

$$
\lim _{\lambda_{0} \downarrow-\infty}\left|\dot{W}\left(\lambda_{n}\right)\right|^{-1}=\lim _{\beta \downarrow-\infty}\left|\dot{W}_{\beta}\left(\lambda_{n}\right)\right|^{-1}=\lim _{\beta \uparrow-\infty}\left|1-\frac{\beta}{\lambda_{n}-\lambda_{0}}\right|^{-1} \cdot\left|\dot{W}\left(\lambda_{n}\right)\right|^{-1}=0 .
$$


Furthermore,

$$
\lim _{\lambda_{0} \uparrow \lambda_{1}}\left|\dot{W}\left(\lambda_{1}\right)\right|^{-1}=\lim _{\beta \uparrow \lambda_{1}-\lambda_{0}}\left|\dot{W}_{\beta}\left(\lambda_{1}\right)\right|^{-1}=\lim _{\beta \uparrow \lambda_{1}-\lambda_{0}}\left|1-\frac{\beta}{\lambda_{1}-\lambda_{0}}\right|^{-1} \cdot\left|\dot{W}\left(\lambda_{1}\right)\right|^{-1}=+\infty .
$$

Let $n=0$. Then

$$
\dot{W}_{\beta}\left(\lambda_{0}+\beta\right)=\frac{W\left(\lambda_{0}+\beta\right)}{\beta}, \quad \frac{\partial \dot{W}\left(\lambda_{0}\right)}{\partial \lambda_{0}}=\left.\frac{\partial}{\partial \beta} \dot{W}_{\beta}\left(\lambda_{0}+\beta\right)\right|_{\beta=0}=\frac{\ddot{W}\left(\lambda_{0}\right)}{2} .
$$

Recall that $\dot{W}\left(\lambda_{0}\right)<0$. Therefore, the Hadamard factorization implies that

$$
W(\lambda) \equiv C\left(\lambda-\lambda_{0}\right) \prod_{n=1}^{+\infty}\left(1-\frac{\lambda-\lambda_{0}}{\lambda_{n}-\lambda_{0}}\right), \quad \lambda \in \mathbb{C},
$$

where $C<0$, whence $\ddot{W}\left(\lambda_{0}\right)=-2 C \sum_{n=1}^{+\infty}\left(\lambda_{n}-\lambda_{0}\right)^{-1}>0$. Thus,

$$
\begin{aligned}
\frac{\partial}{\partial \lambda_{0}}\left|\dot{W}\left(\lambda_{0}\right)\right|^{-1} & =-\frac{\partial}{\partial \lambda_{0}}\left(\dot{W}\left(\lambda_{0}\right)\right)^{-1}=\frac{\ddot{W}\left(\lambda_{0}\right)}{2\left(\dot{W}\left(\lambda_{0}\right)\right)^{2}}>0, \\
\lim _{\lambda_{0} \downarrow-\infty}\left|\dot{W}\left(\lambda_{0}\right)\right|^{-1} & =\lim _{\beta \downarrow-\infty}\left|W_{\beta}\left(\lambda_{0}+\beta\right)\right|^{-1}=\lim _{\beta \downarrow-\infty}|\beta| \cdot\left|W\left(\lambda_{0}+\beta\right)\right|^{-1}=0
\end{aligned}
$$

and

$$
\begin{aligned}
\lim _{\lambda_{0} \uparrow \lambda_{1}}\left|\dot{W}\left(\lambda_{0}\right)\right|^{-1} & =\lim _{\beta \uparrow \lambda_{1}-\lambda_{0}}\left|W_{\beta}\left(\lambda_{0}+\beta\right)\right|^{-1} \\
& =\lim _{\beta \uparrow \lambda_{1}-\lambda_{0}}\left|W\left(\lambda_{0}+\beta\right)\right|^{-1} \cdot|\beta|=+\infty .
\end{aligned}
$$

For each $\left\{\lambda_{n}\right\}_{n=0}^{+\infty}$ and $\left\{\nu_{n}\right\}_{n=0}^{+\infty}$ such that $\lambda_{n}=\lambda_{n}^{0}+c+\mu_{n}, c \in \mathbb{R},\left\{\mu_{n}\right\}_{n=0}^{+\infty} \in \mathcal{M}$ and $\left\{\nu_{n}-\nu_{n}^{0}\right\}_{n=0}^{+\infty} \in \ell_{1}^{2}$, we set

$$
B\left(\lambda_{0}, \lambda_{1}, \ldots ; \nu_{0}, \nu_{1}, \ldots\right):=\sum_{n=0}^{+\infty}\left(2-\frac{e^{\nu_{n}}}{\left|\dot{W}\left(\lambda_{n}\right)\right|}\right) .
$$

Note that this series converges by Theorem 1.1 .

Proof of Corollary 1.2. Fix some $b \in \mathbb{R}$ and $m \geq 0$. By Theorem 1.1, the mapping

$$
q \mapsto \Phi(q, b)=\left(Q_{0}+2 b ;\left\{\mu_{n}(q, b)\right\}_{n=0}^{+\infty} ;\left\{\nu_{n}(q, b)-\nu_{n}^{0}\right\}_{n=0}^{+\infty}\right)
$$

is a bijection between $L^{2}(0,1)$ and the set

$$
\mathcal{S}^{b}=\left\{\left(c ;\left\{\mu_{n}\right\}_{n=0}^{+\infty} ;\left\{\nu_{n}-\nu_{n}^{0}\right\}_{n=0}^{+\infty}\right) \in \mathbb{R} \times \mathcal{M} \times \ell_{1}^{2}: B\left(\lambda_{0}, \lambda_{1}, \ldots ; \nu_{0}, \nu_{1}, \ldots\right)=b\right\} .
$$

Let

$$
P:\left(c ;\left\{\mu_{n}\right\}_{n u=0}^{+\infty} ;\left\{\nu_{n}-\nu_{n}^{0}\right\}_{n=0}^{+\infty}\right) \mapsto\left(c ;\left\{\mu_{n}\right\}_{n=1}^{+\infty} ;\left\{\nu_{n}-\nu_{n}^{0}\right\}_{n=0}^{+\infty}\right) .
$$

Note that $\Phi_{b}(q)=P(\Phi(q, b))$. In particular, $\Phi_{b}$ is real-analytic. Lemma 4.3 gives

$$
\begin{aligned}
\frac{\partial B\left(\lambda_{0}, \ldots ; \nu_{0}, \ldots\right)}{\partial \lambda_{0}}<0, \quad & \lim _{\lambda_{0} \downarrow-\infty} B\left(\lambda_{0}, \ldots ; \nu_{0}, \ldots\right)=+\infty, \\
& \lim _{\lambda_{0} \uparrow \lambda_{1}} B\left(\lambda_{0}, \ldots ; \nu_{0}, \ldots\right)=-\infty .
\end{aligned}
$$

Therefore, for each $\left(c ;\left\{\mu_{n}\right\}_{n=1}^{+\infty} ;\left\{\nu_{n}-\nu_{n}^{0}\right\}_{n=0}^{+\infty}\right) \in \mathbb{R} \times \mathcal{M}^{(1)} \times \ell_{1}^{2}$ there exists a unique $\lambda_{0}^{*}<\lambda_{1}$ such that $B\left(\lambda_{0}^{*}, \lambda_{1}, \ldots ; \nu_{0}, \nu_{1}, \ldots\right)=b$. Thus, the two mappings $P: \mathcal{S}^{b} \rightarrow$ $\mathbb{R} \times \mathcal{M}^{(1)} \times \ell_{1}^{2}$ and $\Phi_{b}: L^{2}(0,1) \rightarrow \mathbb{R} \times \mathcal{M}^{(1)} \times \ell_{1}^{2}$ are bijections. By Theorem 1.1, the mapping

$$
\left(c, \mu_{0}, \nu_{0}, \ldots\right) \mapsto B\left(\lambda_{0}, \lambda_{1}, \ldots ; \nu_{0}, \nu_{1}, \ldots\right)
$$


is real-analytic and $\partial B / \partial \lambda_{0}<0$ (see Lemma 4.3). Now, the implicit function theorem shows that $P^{-1}: \mathbb{R} \times \mathcal{M}^{(1)} \times \ell_{1}^{2} \rightarrow \mathcal{S}^{b}$ is also real-analytic. Therefore, $\left(\Phi^{b}\right)^{-1}$ is realanalytic as a composition of real-analytic mappings.

\section{$\S 5$. Appendix. The case where $\mathbf{a}, \mathbf{b} \in \mathbb{R}$}

Let $\left\{\sigma_{n}(q, a, b)\right\}_{n=0}^{+\infty}$ be the eigenvalues of the Sturm-Liouville problem

$$
-\psi^{\prime \prime}+q(x) \psi=\lambda \psi, \quad \psi^{\prime}(0)-a \psi(0)=0, \quad \psi^{\prime}(1)+b \psi(1)=0,
$$

where $q \in L^{2}(0,1)$ and $a, b \in \mathbb{R}$. It is well known that

$\sigma_{n}(q, a, b)=\sigma_{n}^{0}+Q_{0}+2 a+2 b+\tau_{n}(q, a, b), \quad$ where $\quad\left\{\tau_{n}\right\}_{n=0}^{+\infty} \in \ell^{2} \quad$ and $\quad Q_{0}=\int_{0}^{1} q(t) d t$ (here and below $\sigma_{n}^{0}=\pi^{2} n^{2}, n \geq 0$, denote the unperturbed eigenvalues). Note that $\sigma_{n}(q, a, b)$ are the (simple) roots of the Wronskian

$$
w(\lambda, q, a, b) \equiv\left(\vartheta^{\prime}+a \varphi^{\prime}+b(\vartheta+a \varphi)\right)(1, \lambda, q), \quad \lambda \in \mathbb{C} .
$$

As in [IT83, we introduce the norming constants

$$
\varkappa_{n}(q, a, b)=\log \left[(-1)^{n}(\vartheta+a \varphi)\left(1, \sigma_{n}, q\right)\right]=\log \left|\frac{\psi_{n}(1, q, a, b)}{\psi_{n}(0, q, a, b)}\right|, \quad n \geq 0,
$$

where $\psi_{n}$ is the $n$th normalized eigenfunction such that $\psi_{n}(0)>0$.

Theorem 5.1 (Isaacson, Trubowitz [T83]). The mapping

$$
\Psi:(q ; a ; b) \mapsto\left(Q_{0}+2 a+2 b ;\left\{\tau_{n}(q, a, b)\right\}_{n=0}^{+\infty} ;\left\{\varkappa_{n}(q, a, b)\right\}_{n=0}^{+\infty}\right)
$$

is a real-analytic isomorphism between $L^{2}(0,1) \times \mathbb{R}^{2}$ and $\mathbb{R} \times \mathcal{T} \times \ell_{1}^{2}$, where

$$
\mathcal{T}=\left\{\left\{\tau_{n}\right\}_{n=0}^{+\infty} \in \ell^{2}: \sigma_{0}^{0}+\tau_{0}<\sigma_{1}^{0}+\tau_{1}<\cdots\right\} \subset \ell^{2} .
$$

Remark. This theorem can be reproved much in the same way as we proved Theorem 1.1. In order to consider the case of fixed $a, b \in \mathbb{R}$, we need the following modification of identity (1.4).

Proposition 5.2. For each $(q ; a ; b) \in L^{2}(0,1) \times \mathbb{R}^{2}$, the following identities are valid:

$$
-1+\sum_{n=0}^{+\infty}\left(2-\frac{e^{\varkappa_{n}}}{\left|\dot{w}\left(\sigma_{n}\right)\right|}\right)=b, \quad-1+\sum_{n=0}^{+\infty}\left(2-\frac{e^{-\varkappa_{n}}}{\left|\dot{w}\left(\sigma_{n}\right)\right|}\right)=a
$$

where $w(\lambda)=w(\lambda, q, a, b) \equiv-\sqrt{\lambda} \sin \sqrt{\lambda} \cdot \prod_{n=0}^{+\infty} \frac{\lambda-\sigma_{n}}{\lambda-\sigma_{n}^{0}}$ and $\sigma_{n}=\sigma_{n}(q, a, b), \varkappa_{n}=$ $\varkappa_{n}(q, a, b)$.

Note that (5.2) and Theorem 5.1 immediately give the following well-known result (which generalizes of the Ambarzumyan theorem [Am29]).

Corollary 5.3. Let $a=b \in \mathbb{R}$ and $q \in L_{\text {even }}^{2}(0,1)$, i.e., $q(x) \equiv q(1-x), x \in[0,1]$. Let $p \in L^{2}(0,1)$ be such that $\sigma_{n}(p, a, a)=\sigma_{n}(q, a, a)$ for all $n \geq 0$. Then $p=q$.

Proof. Indeed, we have $w(\lambda, p, a, a) \equiv w(\lambda, q, a, a), \lambda \in \mathbb{C}$, and $\varkappa_{n}(q, a, a)=0$ for all $n \geq 0$. Summing identities (5.2) for the potential $p$ and subtracting the corresponding identities for the potential $q$, we get

$$
\sum_{n=0}^{+\infty} \frac{\cosh \varkappa_{n}(p, a, a)-1}{\left|\dot{w}\left(\sigma_{n}\right)\right|}=0 .
$$

This implies that $\varkappa_{n}(p, a, a)=0$ for all $n \geq 0$. Thus, Theorem 5.1 gives $p=q$. 
Let $a, b \in \mathbb{R}$ be fixed. Then, by (5.2), we can recover $\left(\sigma_{0}, \varkappa_{0}\right)$ by the other spectral data $\left\{\sigma_{n}\right\}_{n=1}^{+\infty},\left\{\varkappa_{n}\right\}_{n=1}^{+\infty}$.

Proposition 5.4. For any $a, b \in \mathbb{R}$, the mapping

$$
\Psi_{a, b}: q \mapsto\left(Q_{0}+2 a+2 b ;\left\{\tau_{n}(q, a, b)\right\}_{n=1}^{+\infty} ;\left\{\varkappa_{n}(q, a, b)\right\}_{n=1}^{+\infty}\right)
$$

is a real-analytic isomorphism between $L^{2}(0,1)$ and $\mathcal{T}^{(1)} \times \ell_{1}^{2}$, where

$$
\mathcal{T}^{(1)}=\left\{\left\{\tau_{n}\right\}_{n=1}^{+\infty} \in \ell^{2}: \sigma_{1}^{0}+\tau_{1}<\sigma_{2}^{0}+\tau_{2}<\cdots\right\} \subset \ell^{2} .
$$

Proof of Proposition 5.2. We prove the first identity in (5.2); the proof of the second is similar. Consider the meromorphic function

$$
f(\lambda):=\frac{(\vartheta+a \varphi)(1, \lambda, q)}{w(\lambda, q, a, b)} \equiv \frac{\vartheta+a \varphi}{\left(\vartheta^{\prime}+a \varphi^{\prime}\right)+b(\vartheta+a \varphi)}(1, \lambda, q), \quad \lambda \in \mathbb{C} .
$$

All roots $\sigma_{n}=\sigma_{n}(q, a, b)$ of $w(\lambda)$ are simple. The definition (5.1) shows that

$$
\underset{\lambda=\sigma_{n}}{\operatorname{res}} f(\lambda)=\frac{(\vartheta+a \varphi)(1, \lambda, q)}{\dot{w}\left(\sigma_{n}\right)}=\frac{(-1)^{n} e^{\varkappa_{n}}}{\dot{w}\left(\sigma_{n}\right)}=-\frac{e^{\varkappa_{n}}}{\left|\dot{w}\left(\sigma_{n}\right)\right|} .
$$

Put $|\lambda|=\pi^{2}\left(m+\frac{1}{2}\right)^{2} \rightarrow \infty$. Due to the asymptotics (2.2)-(2.5), we have

$$
\begin{aligned}
f(\lambda) & =\frac{\cos \sqrt{\lambda}+\lambda^{-1 / 2} \sin \sqrt{\lambda} \cdot\left(\frac{1}{2} Q_{0}+a\right)+o\left(\lambda^{-1 / 2} e^{|\operatorname{Im} \sqrt{\lambda}|}\right)}{-\lambda^{1 / 2} \sin \sqrt{\lambda}+\cos \sqrt{\lambda} \cdot\left(\frac{1}{2} Q_{0}+a+b\right)+o\left(e^{|\operatorname{Im} \sqrt{\lambda}|}\right)} \\
& =-\lambda^{-\frac{1}{2}} \cot \lambda-\lambda^{-1} \cdot\left(\frac{1}{2} Q_{0}+a\right)-\lambda^{-1} \cot ^{2} \lambda \cdot\left(\frac{1}{2} Q_{0}+a+b\right)+o\left(\lambda^{-1}\right) .
\end{aligned}
$$

Let $f_{0}(\lambda) \equiv-\lambda^{\frac{-12}{\cot }} \sqrt{\lambda}$. Applying the Cauchy theorem in the disk $|\lambda|<\pi^{2}\left(m+\frac{1}{2}\right)^{2}$, we see that

$$
\sum_{n=0}^{m}\left(\underset{\lambda=\sigma_{n}}{\operatorname{res}} f(\lambda)-\underset{\lambda=\sigma_{n}^{0}}{\operatorname{res}} f_{0}(\lambda)\right)=-\left(\frac{1}{2} Q_{0}+a\right)-\left(\frac{1}{2} Q_{0}+a+b\right) \cdot \sum_{n=0}^{m} \underset{\lambda=\sigma_{n}^{0}}{\operatorname{res}}\left(f_{0}(\lambda)\right)^{2} .
$$

Note that

$$
f_{0}(\lambda)=-\frac{1}{\sqrt{\lambda} \tan \sqrt{\lambda}}=-\frac{1}{\lambda}\left(1-\frac{\lambda}{3}+O\left(\lambda^{2}\right)\right) \quad \text { as } \quad \lambda \rightarrow \sigma_{0}=0,
$$

and

$$
f_{0}(\lambda)=-\frac{2}{\lambda-\sigma_{n}^{0}}\left(1-\frac{\lambda-\sigma_{n}^{0}}{4 \sigma_{n}^{0}}+O\left(\left(\lambda-\sigma_{n}^{0}\right)^{2}\right)\right) \quad \text { as } \quad \lambda \rightarrow \sigma_{n}^{0}, \quad n \geq 1 .
$$

Hence,

$$
\begin{array}{cl}
\underset{\lambda=\sigma_{0}^{0}}{\operatorname{res}} f_{0}(\lambda)=-1, & \underset{\lambda=\sigma_{n}^{0}}{\operatorname{res}} f_{0}(\lambda)=-2, \quad n \geq 1, \\
\underset{\lambda=\sigma_{0}^{0}}{\operatorname{res}}\left(f_{0}(\lambda)\right)^{2}=-\frac{2}{3}, & \underset{\lambda=\sigma_{n}^{0}}{\operatorname{res}}\left(f_{0}(\lambda)\right)^{2}=-\frac{2}{\sigma_{n}^{0}}, \quad n \geq 1,
\end{array}
$$

and

$$
\sum_{n=0}^{+\infty} \underset{\lambda=\sigma_{n}^{0}}{\operatorname{res}}\left(f_{0}(\lambda)\right)^{2}=-\frac{2}{3}-\frac{2}{\pi^{2}} \sum_{n=0}^{+\infty} \frac{1}{n^{2}}=-\frac{2}{3}-\frac{2}{\pi^{2}} \cdot \frac{\pi^{2}}{6}=-1 .
$$

Substituting these identities and (5.3) in (5.4), we obtain (5.2) as $m \rightarrow \infty$. 
For each $\left\{\sigma_{n}\right\}_{n=0}^{+\infty}$ such that $\sigma_{n}=\sigma_{n}^{0}+c+\tau_{n}, c \in \mathbb{R},\left\{\tau_{n}\right\}_{n=0}^{+\infty} \in \mathcal{T}$ and $\left\{\varkappa_{n}\right\}_{n=0}^{+\infty} \in \ell_{1}^{2}$, we denote

$$
\begin{aligned}
W(\lambda) & =W\left(\lambda ; \sigma_{0}, \sigma_{1}, \ldots\right):=-\sqrt{\lambda} \cos \sqrt{\lambda} \cdot \prod_{k=0}^{+\infty} \frac{\lambda-\sigma_{k}}{\lambda-\sigma_{k}^{0}}, \quad \lambda \in \mathbb{C}, \\
F\left(\sigma_{0}\right) & =F\left(\sigma_{0}, \sigma_{1}, \ldots\right):=\frac{1}{\left|\dot{W}\left(\sigma_{0}\right)\right|}, \\
G_{ \pm}\left(\sigma_{0}\right) & =G_{ \pm}\left(\sigma_{0}, \sigma_{1}, \ldots ; \varkappa_{1}, \varkappa_{2}, \ldots\right):=-1+\sum_{n=1}^{+\infty}\left(\frac{e^{ \pm \varkappa_{n}}}{\left|\dot{W}\left(\sigma_{n}\right)\right|}-2\right)
\end{aligned}
$$

(the series converge by Theorem 5.1 and Proposition 5.2). Using this notation, we rewrite (5.2) in the following form:

$$
e^{-\varkappa_{0}} F\left(\sigma_{0}\right)+G_{-}\left(\sigma_{0}\right)=-a, \quad e^{\varkappa_{0}} F\left(\sigma_{0}\right)+G_{+}\left(\sigma_{0}\right)=-b .
$$

Lemma 5.5. (i) Suppose $c \in \mathbb{R},\left\{\tau_{n}\right\}_{n=0}^{+\infty} \in \mathcal{T}$, and $\left\{\varkappa_{n}\right\}_{n=0}^{+\infty} \in \ell_{1}^{2}$. Then

$$
\begin{array}{lll}
F^{\prime}\left(\sigma_{0}\right)>0, & \lim _{\sigma_{0} \downarrow-\infty} F\left(\sigma_{0}\right)=0, & \lim _{\sigma_{0} \uparrow \sigma_{1}} F\left(\sigma_{0}\right)=+\infty ; \\
G_{ \pm}^{\prime}\left(\sigma_{0}\right)>0, & \lim _{\sigma_{0} \downarrow-\infty} G_{ \pm}\left(\sigma_{0}\right)=-\infty, & \lim _{\sigma_{0} \uparrow \sigma_{1}} G_{ \pm}\left(\sigma_{0}\right)=+\infty .
\end{array}
$$

(ii) For each $a, b, c \in \mathbb{R},\left\{\tau_{n}\right\}_{n=1}^{+\infty} \in \mathcal{T}^{(1)}$, and $\left\{\varkappa_{n}\right\}_{n=1}^{+\infty} \in \ell_{1}^{2}$, there exist unique $\sigma_{0}<\sigma_{1}$ and $\varkappa_{0} \in \mathbb{R}$ such that identities (5.5) (or, equivalently, (5.2)) hold true.

Proof. (i) The proof repeats that of Lemma 4.3 .

(ii) Identities (5.5) are equivalent to

$$
\left(F\left(\sigma_{0}\right)\right)^{2}=\left(a+G_{-}\left(\sigma_{0}\right)\right)\left(b+G_{+}\left(\sigma_{0}\right)\right)
$$

and

$$
e^{\varkappa_{0}}=-\frac{F\left(\sigma_{0}\right)}{a+G_{-}\left(\sigma_{0}\right)}=-\frac{b+G_{+}\left(\sigma_{0}\right)}{F\left(\sigma_{0}\right)} .
$$

Note that $F\left(\sigma_{0}\right)>0$ for all $\sigma_{0}<\sigma_{1}$. Relations (5.7) imply that there exist unique values $\sigma_{ \pm}^{*}<\sigma_{1}$ such that $a+G_{-}\left(\sigma_{-}^{*}\right)=b+G_{+}\left(\sigma_{+}^{*}\right)=0$. Let $\sigma_{0}^{*}=\min \left(\sigma_{-}^{*}, \sigma_{+}^{*}\right)$ and

$$
G\left(\sigma_{0}\right):=\left(a+G_{-}\left(\sigma_{0}\right)\right) \cdot\left(b+G_{+}\left(\sigma_{0}\right)\right), \quad \sigma_{0} \leq \sigma_{0}^{*} .
$$

Using (5.7), it is easy to conclude that

$$
G^{\prime}\left(\sigma_{0}\right)<0, \quad \lim _{\sigma_{0} \downarrow-\infty} G\left(\sigma_{0}\right)=+\infty, \quad G\left(\sigma_{0}^{*}\right)=0,
$$

while

$$
\left(F^{2}\right)^{\prime}\left(\sigma_{0}\right)>0, \quad \lim _{\sigma_{0} \downarrow-\infty}\left(F\left(\sigma_{0}\right)\right)^{2}=0, \quad\left(F\left(\sigma_{0}^{*}\right)\right)^{2}>0 .
$$

Therefore, the equation $\left(F\left(\sigma_{0}\right)\right)^{2}=G\left(\sigma_{0}\right)$ has a unique solution $\sigma_{0}<\sigma_{0}^{*}$ (and has no solutions $\sigma_{0} \geq \sigma_{0}^{*}$ due to a simple consideration of signs), and $\varkappa_{0}$ is determined by $\tau_{0}$.

Proof of Proposition 5.4. Fix some $a, b \in \mathbb{R}$ and $m \geq 0$. By Theorem 5.1 and Proposition 5.2. the mapping

$$
q \mapsto \Psi(q, a, b)=\left(Q_{0}+2 a+2 b ;\left\{\tau_{n}(q, a, b)\right\}_{n=0}^{+\infty} ;\left\{\varkappa_{n}(q, a, b)\right\}_{n=0}^{+\infty}\right)
$$

is a bijection between $L^{2}(0,1)$ and the set

$$
\mathcal{S}^{a, b}=\left\{\left(c ;\left\{\tau_{n}\right\}_{n=0}^{+\infty} ;\left\{\varkappa_{n}\right\}_{n=0}^{+\infty}\right) \in \mathbb{R} \times \mathcal{T} \times \ell_{1}^{2}: e^{-\varkappa_{0}} F+G_{-}=-a, \quad e^{\varkappa_{0}} F+G_{+}=-b\right\} .
$$


Let

$$
P:\left(c ;\left\{\tau_{n}\right\}_{n=0}^{+\infty} ;\left\{\varkappa_{n}\right\}_{n=0}^{+\infty}\right) \mapsto\left(c ;\left\{\tau_{n}\right\}_{n=1}^{+\infty} ;\left\{\varkappa_{n}\right\}_{n=1}^{+\infty}\right) .
$$

Note that $\Psi_{a, b}(q)=P(\Psi(q, a, b))$. In particular, $\Psi_{a, b}$ is real-analytic. By Lemma 5.5 (ii), for each $\left(c ;\left\{\tau_{n}\right\}_{n=1}^{+\infty} ;\left\{\varkappa_{n}\right\}_{n=1}^{+\infty}\right) \in \mathbb{R} \times \mathcal{M}^{(1)} \times \ell_{1}^{2}$ there exist unique $\sigma_{0}<\sigma_{1}$ and $\varkappa_{0} \in \mathbb{R}$ such that identities (5.5) hold true. Therefore, the mappings $P: \mathcal{S}^{a, b} \rightarrow \mathbb{R} \times \mathcal{M}^{(1)} \times \ell_{1}^{2}$ and $\Psi_{a, b}: L^{2}(0,1) \rightarrow \mathbb{R} \times \mathcal{M}^{(1)} \times \ell_{1}^{2}$ are bijections.

By Theorem 5.1

$$
\left(c ;\left\{\tau_{n}\right\}_{n=0}^{+\infty} ;\left\{\varkappa_{n}\right\}_{n=0}^{+\infty}\right) \mapsto(-a ;-b)=\left(e^{-\varkappa_{0}} F\left(\sigma_{0}\right)+G_{-}\left(\sigma_{0}\right) ; e^{\varkappa_{0}} F\left(\sigma_{0}\right)+G_{+}\left(\sigma_{0}\right)\right)
$$

is a real-analytic mapping. By Lemma 5.5, $\frac{\partial a}{\partial \sigma_{0}}<0, \frac{\partial b}{\partial \sigma_{0}}<0, \frac{\partial a}{\partial \varkappa_{0}}=e^{-\varkappa_{0}} F>0$, and $\frac{\partial b}{\partial \varkappa_{0}}=e^{\varkappa_{0}} F<0$. Therefore,

$$
\operatorname{det}\left(\begin{array}{ll}
\partial a / \partial \sigma_{0} & \partial b / \partial \sigma_{0} \\
\partial a / \partial \varkappa_{0} & \partial b / \partial \varkappa_{0}
\end{array}\right)>0
$$

So, $P^{-1}: \mathbb{R} \times \mathcal{T}^{(1)} \times \ell_{1}^{2} \rightarrow \mathcal{S}^{a, b}$ is real-analytic, by the implicit function theorem. Thus, $\Psi_{a, b}^{-1}$ is also real-analytic, being the composition of real-analytic mappings.

\section{ACKNOWLEDGMENTS}

The greater part of this paper was written during the authors' stay at the MittagLeffler Institute, Djursholm (program "Wave Motion", fall 2005). The authors are grateful to the Institute for its hospitality. Also, the authors would like to thank Professor Markus Klein for useful discussions.

\section{REFERENCES}

[Am29] V. A. Ambarzumyan, Über eine Frage der Eigenwerttheorie, Z. Phys. 53 (1929), 690-695.

[Bo46] G. Borg, Eine Umkehrung der Sturm-Liouvilleschen Eigenwertaufgabe. Bestimmung der Differentialgleichung durch die Eigenwerte, Acta Math. 78 (1946), 1-96. MR0015185 (7:382d)

[CK07] D. Chelkak and E. Korotyaev, The inverse problem for perturbed harmonic oscillator on the half-line with a Dirichlet boundary condition, Ann. Henri Poincaré 8 (2007), 1115-1150. MR2355343 (2008m:34012)

[CKK04] D. Chelkak, P. Kargaev, and E. Korotyaev, Inverse problem for harmonic oscillator perturbed by potential, characterization, Comm. Math. Phys. 249 (2004), 133-196. MR.2077254 (2005h:81120)

[DT84] B. Dahlberg and E. Trubowitz, The inverse Sturm-Liouville problem. III, Comm. Pure Appl. Math. 37 (1984), 255-267. MR0733718 (86f:34052)

[GL51] I. M. Gel'fand and B. M. Levitan, On the determination of a differential equation from its spectral function, Izv. Akad. Nauk SSSR. Ser. Mat. 15 (1951), no. 4, 309-360. (Russian) MR0045281(13:558f)

[IT83] E. Isaacson and E. Trubowitz, The inverse Sturm-Liouville problem. I, Comm. Pure Appl. Math. 36 (1983), 767-783. MR0720593 (85d:34024)

[IMT84] E. Isaacson, H. P. McKean, and E. Trubowitz, The inverse Sturm-Liouville problem. II, Comm. Pure Appl. Math. 37 (1984), 1-11. MR0728263 (86f:34051)

[JL97] M. Jodeit and B. M. Levitan, The isospectrality problem for the classical Sturm-Liouville equation, Adv. Differential Equations 2 (1997), 297-318. MR.1424771(97i:34036)

[Lev84] B. M. Levitan, Inverse Sturm-Liouville problems, Nauka, Moscow, 1984; English transl., VSP, Zeist, 1987. MR0771843 (86d:34002) MR0933088 (89b:34001)

[Mar77] V. A. Marchenko, Sturm-Liouville operators and their applications, Naukova Dumka, Kiev, 1977; English transl., Oper. Theory Adv. Appl., vol. 22, Birkhäuser Verlag, Basel, 1986. MR0481179 (58:1317); MR0897106 (88f:34034) 
[PT87] J. Pöschel and E. Trubowitz, Inverse spectral theory, Pure Appl. Math., vol. 130, Acad. Press, Inc., Boston, MA, 1987. MR0894477 (89b:34061)

[CK09] D. Chelkak and E. Korotyaev, Weyl-Titchmarsh functions of vector-valued Sturm-Liouville operators on the unit interval, J. Funct. Anal. 257 (2009), 1546-1588. MR2541280

School of Mathematics, Cardiff University, Senghennydd Road, CF24 4AG Cardiff, Wales, UNITED KINGDOM

E-mail address: korotyaev@gmail.com

Department of Mathematics and Mechanics, St. Petersburg State University, UniverSitetskit Prospekt 28, Staryi Petergof, St. Petersburg 198504, Russia

E-mail address: dchelkak@pdmi.ras.ru

Received 15/MAR/2008

Translated by THE AUTHORS 